\title{
MTOR Kinase is a Therapeutic Target for Respiratory Syncytial Virus and Coronaviruses
}

\section{HoangDinh Huynh}

The University of Texas Southwestern Medical Center

\section{Ruth Levitz}

The University of Texas Southwestern Medical Center

Jeffrey Kahn ( $\nabla$ Jeffrey.Kahn@UTSouthwestern.edu )

The University of Texas Southwestern Medical Center

\section{Research Article}

Keywords: Therapeutic interventions, MTOR Kinase, Respiratory Syncytial Virus, Coronaviruses

Posted Date: May 28th, 2021

DOI: https://doi.org/10.21203/rs.3.rs-537263/v1

License: (1) This work is licensed under a Creative Commons Attribution 4.0 International License.

Read Full License

Version of Record: A version of this preprint was published at Scientific Reports on December 1st, 2021.

See the published version at https://doi.org/10.1038/s41598-021-03814-7. 
mTOR Kinase is a Therapeutic Target for Respiratory Syncytial Virus and Coronaviruses

HoangDinh Huynh ${ }^{1}$, Ruth Levitz ${ }^{1}$, and Jeffrey S. Kahn ${ }^{1,2}$

${ }^{1}$ Department of Pediatrics and ${ }^{2}$ Department of Microbiology University of Texas Southwestern Medical Center, Dallas, Texas 75390 USA

Running Title: Replication of Respiratory Syncytial Virus and Coronaviruses are impeded by mTOR Inhibitors

Corresponding author: Jeffrey S. Kahn (Jeffrey.Kahn@UTSouthwestern.edu) 


\section{SUMMARY}

Therapeutic interventions targeting viral infections remain a significant challenge for both the medical and scientific communities. While specific antiviral agents have shown success as therapeutics, viral resistance inevitably develops making many of these approaches ineffective. This inescapable obstacle warrants alternative approaches, such as targeting host cellular factors. Respiratory syncytial virus (RSV), the major respiratory pathogen of infants and children worldwide, causes respiratory tract infection ranging from mild upper respiratory tract symptoms to severe life-threatening lower respiratory tract disease. Despite the fact that the molecular biology of the virus, which was originally discovered in 1956, is well described, there is no vaccine or effective antiviral treatment against RSV infection. Here, we demonstrate that targeting host factors, specifically, mTOR signaling, limits RSV protein production and viral replication. Further, we show that this approach is generalizable as inhibition of mTOR kinases reduces coronavirus gene expression, protein production and replication. Overall, defining virus replicationdependent host functions may be an effective means to combat viral infections, particular in the absence of antiviral drugs.

\section{HIGHLIGHTS}

- Dynamic utilization of mTOR signaling by RSV for its replication.

- The redundancy of mTOR complexes for RSV replication.

- The clinical implication of mTOR and Akt inhibitors on RSV replication and cancer patients.

- mTOR kinase is a therapeutic target for coronavirus. 


\section{INTRODUCTION}

Respiratory syncytial virus (RSV) is the major respiratory pathogen of infants and children worldwide ${ }^{1}$. Globally, RSV accounts $\sim 200,000$ deaths per year in children less than 5 years old making RSV the $3^{\text {rd }}$ most common cause of death secondary to pneumonia in

this age group (after Streptococcus pneumoniae and Haemophilus influenza) ${ }^{2}$. The clinical manifestations of RSV infection in young children range from mild upper respiratory tract symptoms to severe life-threatening lower respiratory tract disease. The epidemiology of RSV is complex and dynamic. RSV strains can be classified, based on serological and genetic methods, into 1 of 2 subgroups (A or B) and both subgroup $A$ and $B$ viruses circulate during each seasonal epidemic ${ }^{3,4}$. Strains vary from year to year and strains identified in one location may be similar to strains identified in different years in vastly remote geographic locations.

RSV is a single-stranded, negative sense, non-segmented enveloped RNA virus ${ }^{5}$. The genome is $\sim 15,000$ bases and encodes at least 11 genes, some of which are encoded in overlapping reading frames and the intergenic distances are relatively short ${ }^{6}$. The genome encodes three virion glycoproteins: fusion $(F)$, attachment $(G)$, and short hydrophobic $(\mathrm{SH})^{7}$. The $\mathrm{F}$ protein is an essential component of the virus and is responsible for viral attachment and the classic "syncytia" observed in cell culture ${ }^{8}$. The nucleocapsid $(\mathrm{N})$, and matrix $(\mathrm{M})$ encode structural proteins of the virion and tend to be highly conserved among isolates. RSV replicates entirely in the cytoplasm without involvement of the nucleus ${ }^{9}$ and can replicated in cells devoid of nuclei ${ }^{10}$.

The management of RSV has changed little since it was first isolated from symptomatic children in $1957{ }^{11}$ (the virus was originally discovered in chimpanzees in $1956{ }^{12}$ ). There 
is no effective vaccine to protect against RSV infection. Antiviral therapy is of limited effectiveness, at best, and is rarely used in the management of RSV infection ${ }^{13}$. The treatment of the RSV infected child is essentially supportive care (supplemental oxygen, hydration, invasive or non-invasive respiratory support if necessary). A humanized monoclonal antibody (palivizumab) was approved by the FDA in 1998 to protect high risk infants against severe RSV disease, however this product is not approved for use in the vast majority of otherwise healthy infants and does not protect against RSV infection; in addition, this product has no therapeutic effectiveness ${ }^{14}$.

At present, there are several promising antiviral drugs in development and some have advanced to clinical trials. These agents can be classified into four categories: immunoglobulins, nucleoside analogues, small interfering RNAs, and fusion inhibitors (small molecules) ${ }^{15,16}$. These drugs were developed to target virus-specific molecules and functions. Although these drugs are promising, one foreseeable obstacle is the development of viral resistance, as genomic mutations occur during viral replication (this is true for all RNA viruses) ${ }^{17,18}$. Therefore, alternative therapeutic approaches should be considered.

RSV utilizes specific host intracellular pathway(s) for viral replication and viral protein synthesis ${ }^{18}$ and these pathways are potential targets for antiviral therapy. Therefore, we hypothesized that interfering with specific host cellular function(s) required for RSV protein synthesis would mitigate or eliminate viral growth, regardless of viral mutations. There are many host cellular processes that involve protein synthesis and several of these pathways are potential targets for cancer therapeutics. One pathway is the mechanistic target of rapamycin (mTOR) signaling, known to regulate many fundamental 
cellular processes, including protein synthesis ${ }^{19}$. mTOR is a serine/threonine protein kinase that belongs to the phosphoinositide 3-kinase (PI3K)-related kinase family ${ }^{19}$. mTOR kinase interacts with several proteins to form two distinct complexes, known as mTOR complex 1 (mTORC1) and mTOR complex 2 (mTORC2). mTORC1 contains the regulatory subunit Raptor, which is sensitive to rapamycin, while mTORC2, which contains Rictor, is rapamycin-insensitive. Aside from protein synthesis, mTOR signaling plays a key role in many fundamental cellular processes, including energy metabolism, lipogenesis, autophagy, lysosome biogenesis, cytoskeletal organization, and cell growth and survival ${ }^{19}$. To evaluate the role of mTOR complexes in RSV replication, we used various clinical isolates of RSV previously characterized ${ }^{20-22}$.

To address whether this approach in which mTOR-associated pathways are specifically targeted could be exploited to inhibit replication of viruses other than RSV, we choose to use our system to examine the effect of mTOR kinase inhibition on coronaviruses given the current COVID-19 pandemic. Endemic common human coronaviruses (HCoVs), such as 229E, NL-63, OC43, and HKU-1 are responsible for mild respiratory illnesses like the common cold ${ }^{23}$ though these viruses can cause severe or life-threatening disease ${ }^{24}$. In the last 2 decades, 3 highly pathogenic human coronaviruses have emerged: severe acute respiratory syndrome coronavirus 1 (SARS-CoV-1) (2002) ${ }^{25}$, Middle East respiratory syndrome coronavirus (MERS-CoV) (2012) ${ }^{26}$, and the severe acute respiratory syndrome coronavirus 2 (SARS-CoV-2) (2019), now known to cause current pandemic disease COVID-19 ${ }^{27}$. HCoV 229E and NL-63 belong to genus alphacoronavirus, whereas HCoV OC43, HKU-1, SARS-CoV-1, MERS, and SARS-CoV-2 are belong to beta-coronavirus. There is currently a great need for effective strategies to treat 
HCoV infections. Here we show that targeting mTOR, as an antiviral approach, is generalizable and may be an effective strategy against highly pathogenic coronaviruses such as SARS-CoV-1, MERS and SARS-CoV-2.

\section{RESULTS}

Pharmacologic inhibition of mTORC1 increases viral protein synthesis and replication

We have previously characterized RSV clinical isolates in terms of severity of illness ${ }^{22}$ and induction of the innate immune system ${ }^{21}$. Among the subgroup $A$ and $B$ strains selected for this study, there was a varying degree of viral protein synthesis (Supplementary Figure 1A-C). From these, we arbitrarily selected NH409A, a subgroup A strain, to evaluate whether mTORC1 has any significant role in RSV replication. A549 cells were infected with RSV strain NH409A for 1.5 hours ${ }^{20,22}$. Infected A549 cells were then treated at varying doses with rapamycin (sirolimus), mTORC1-specific inhibitor 28-30 for 22.5 hours. Western blotting and plaque titration assay were performed at 24 hours post-infection. At low dose $(0.1 \mathrm{nM})$, rapamycin completely inhibited S6K1 phosphorylation, a read-out for mTORC1 activity and this correlated with the activation (phosphorylation) of Akt, a well-known downstream target for mTORC2 (Figure 1A-C). Consistence with the reduced level of S6K1 phosphorylation, rapamycin treatment predictably decreased phosphorylation of ribosomal protein S6 (upper band) (Figure 1A \& D). Inhibition of mTORC1 by rapamycin resulted a statistically significant induction of viral nucleocapsid $\mathrm{N}$ and fusion $\mathrm{F} 1$ proteins (Figure 1E-F). Functionally, elevated viral 
protein levels also correlated with statistically significant increase in viral replication and production of virus progeny (Figure 1G). Further, induction of viral proteins and replication was observed at high doses of rapamycin (10-100 nM) (Supplementary Figure 2A-E), though the maximal effect appears to be in the range of 1-10 nM. These observations were replicated with $\mathrm{NH} 1101 \mathrm{~A}$, a subgroup A3 RSV isolate (Supplementary Figure 3AD), and $\mathrm{NH} 1125 \mathrm{~B}$, a B subgroup RSV isolate (Supplementary Figure 4A-I) indicating that the effect of rapamycin is generalizable across RSV strains. These initial observations warranted a further mechanistic study of how inhibition of mTORC1 signaling induced RSV replication.
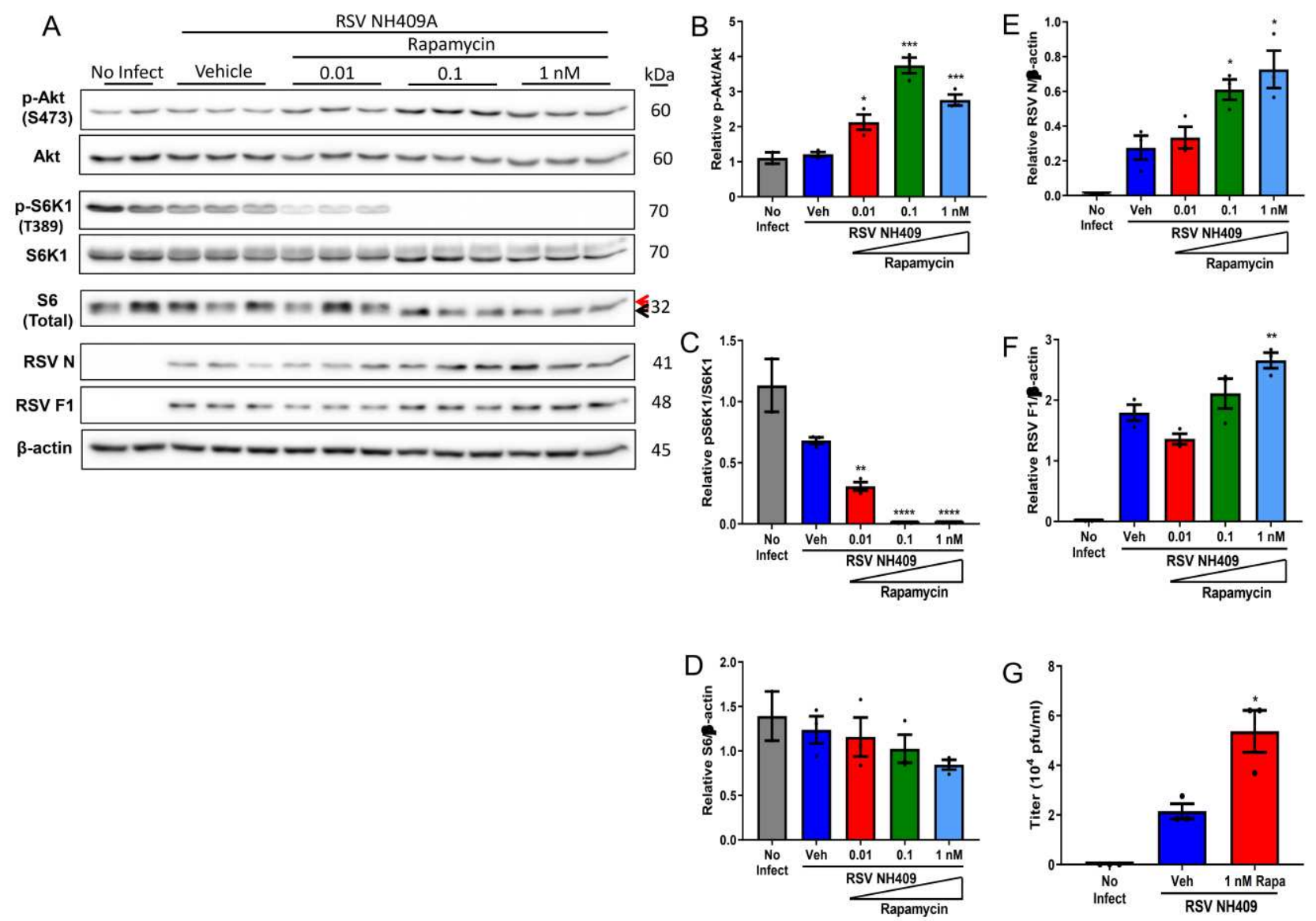

Figure 1: Rapamycin enhances viral protein expression and replication. 
A549 cells were infected with clinical isolate NH409A and subsequently treated with varying concentrations of rapamycin. Protein analyses and viral replication was measured at 24 hours post infection (m.o.i $=0.2$ ).

(A) Western blot of cellular and viral proteins. The 2 forms of S6 are designated by the red and black arrows.

(B-F) Quantification of cellular and viral proteins displayed in A $(n=3)$.

(B) phospho-Akt/Akt; (C) phospho-S6K1/S6K1; (D) total ribosomal protein S6/ $\beta$ actin; (E) RSV nucleoprotein N/ $\beta$-actin; (F) RSV fusion protein $\mathrm{F} 1 / \beta$-actin.

(G) Quantification of virus progeny in the absence and presence of rapamycin $(n=3)$. Error bars, SEM; ${ }^{*}, \mathrm{p}<0.05 ;{ }^{* *}, \mathrm{p}<0.01 ;{ }^{* * *}, \mathrm{p}<0.005 ;{ }^{* * *}, \mathrm{p}<0.001$; n.s. non-significant.

\section{Akt inhibitor enhances viral protein synthesis and replication}

The inhibition of mTORC1 with rapamycin correlated with the predictable activation (phosphorylation) of Akt (Figure 1A-B), due to the loss of negative feedback loops ${ }^{31,32}$. Akt, an upstream activator of mTORC1, is known to promote cell survival, proliferation, and growth ${ }^{19,33,34}$. Activation of Akt is also known to be involved in many cancers ${ }^{35}$. Our data suggested that the increased activation of Akt correlated with the induction of viral protein synthesis and viral replication. To determine whether Akt has any impact on RSV replication, infected A549 cells were treated with MK-2206, a known pan-Akt inhibitor ${ }^{36}$. Treatment with MK-2206 abolished Akt phosphorylation at $1 \mu \mathrm{M}$, which correlated with reduction of S6K1 activation, and total S6 (Figure 2A-D). Inactivation of Akt correlated with modest enhancement of viral protein synthesis and the production of virus progeny (Figure 2E-G), consistent with the findings following rapamycin treatment (Figure 1). Our observations suggested a potential similar impact of other class of Akt inhibitors on RSV replication. 

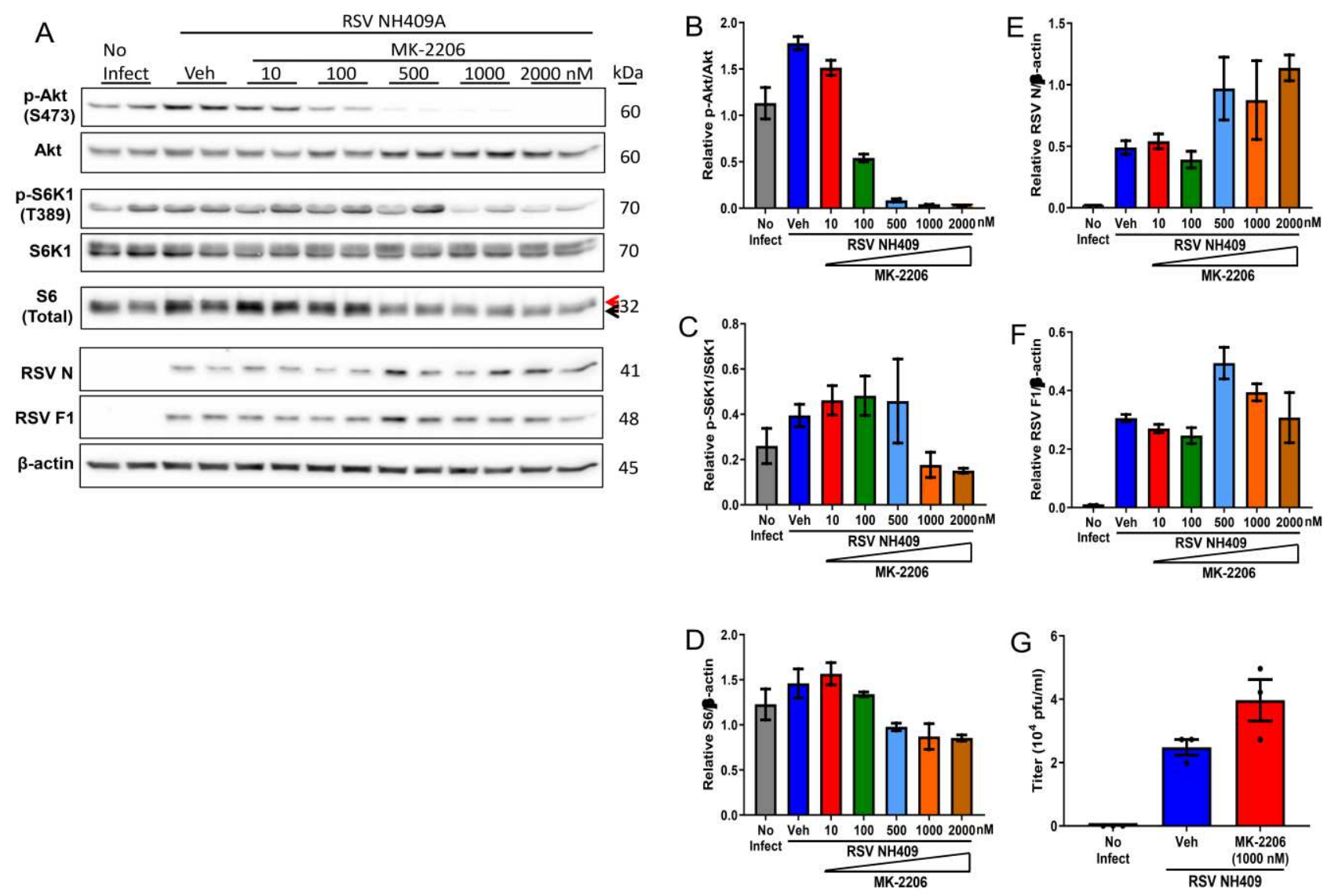

Figure 2: Akt inhibitor enhances viral proteins and replication.

A549 cells were infected with clinical isolate $\mathrm{NH} 409 \mathrm{~A}$ and subsequently treated with varying concentrations of MK-2206, an Akt inhibitor. Protein analyses and viral replication was measured at 24 hours post infection (m.o.i $=0.2$ ).

(A) Western blot of cellular and viral proteins. The 2 forms of S6 are designated by the red and black arrows.

(B-F) Quantification of cellular and viral proteins displayed in $A(n=2)$.

(B) phospho-Akt/Akt; (C) phospho-S6K1/S6K1; (D) total ribosomal protein S6/ßactin; (E) RSV nucleoprotein N/ $\beta$-actin; (F) RSV fusion protein F1/ $\beta$-actin.

(g) Quantification of virus progeny in the absence and presence of MK-2206 $(n=3)$. Error bars, SEM; ${ }^{*}, p<0.05 ;{ }^{* *}, p<0.01 ;{ }^{* * *}, p<0.005 ;{ }^{* \star *}, p<0.001$; n.s. non-significant.

\section{mTORC1 and mTORC2 provide redundant activities for RSV replication}

Treatment with Akt inhibitor MK-2206 (downstream of mTORC2) enhanced viral replication, suggesting mTORC2 may be involved in viral replication. mTORC1 contains 
subunit Raptor, while mTORC2 contains Rictor ${ }^{19}$. Due to lack of proven mTORC2specific inhibitors, we used a genetic approach to define the role of mTORC2 in RSV replication. Raptor or Rictor was genetically knockdown by stable lenti-viral short-hairpin RNA (shRNA) (Figure 3A \& Supplementary Figure 5) ${ }^{37}$. The knockdown of Raptor significantly reduced S6K1 phosphorylation, while elevating Akt phosphorylation (Figure 3B-E), which is consistent with rapamycin treatment (Figure 1). In similar fashion, the knockdown of Rictor significant reduced Akt phosphorylation, while elevating S6K1 phosphorylation. Knockdown of either Raptor or Rictor correlated with increased viral protein synthesis (Figure 3F-G). These observations suggest mTORC1 and mTORC2 has a redundant role for RSV replication.
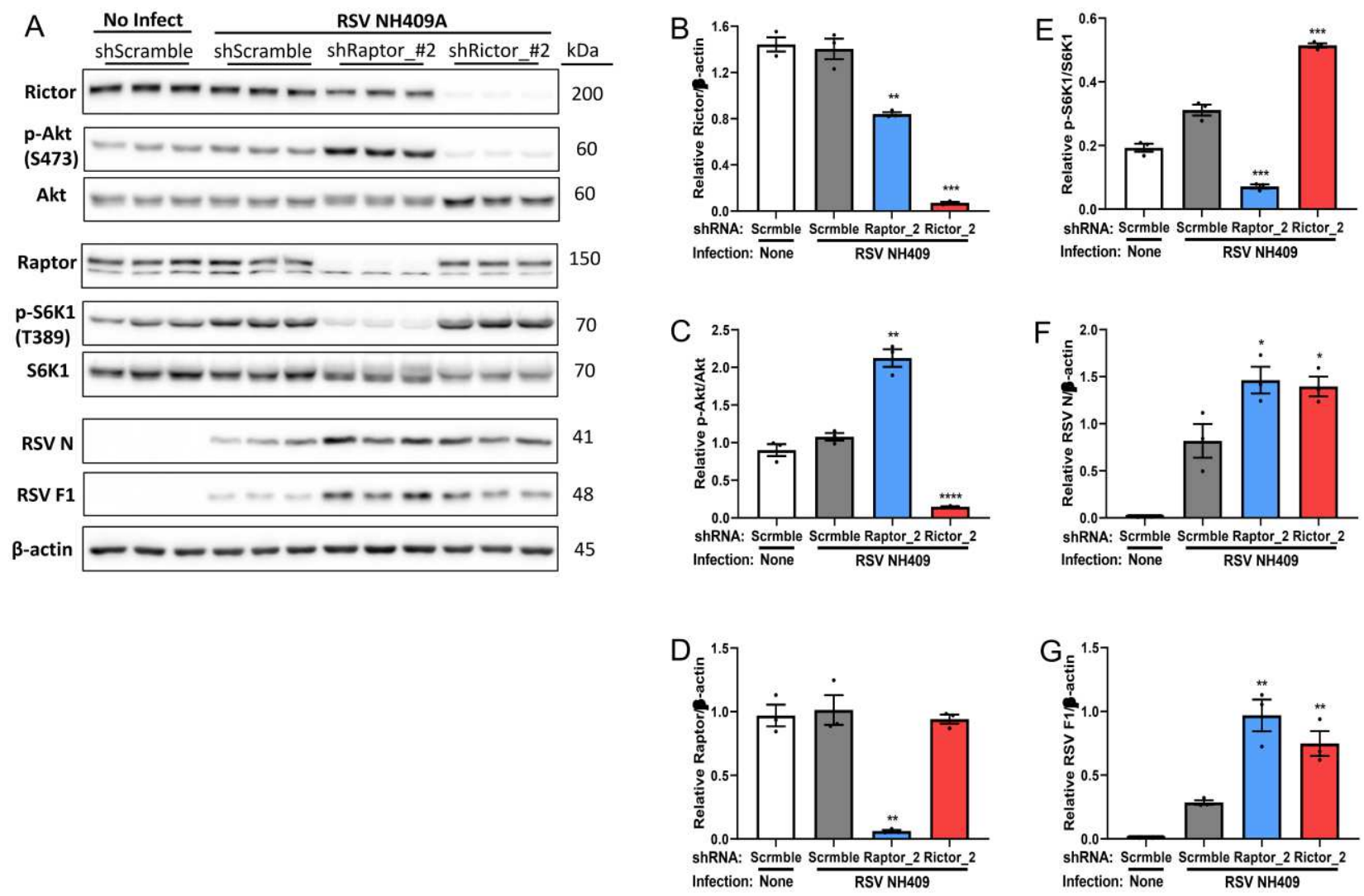

Figure 3: Genetic knockdown of Raptor or Rictor increases RSV protein synthesis.

Lenti-viral short-hairpin RNA (shRNA) were used to knockdown expression of either Raptor or Rictor in A549 cells. shRaptor- or shRictor-A549 cells were infected with RSV 
$\mathrm{NH} 409 \mathrm{~A}$. Cellular and viral protein expression was measures at 24 hour post infection (m.o.i = 0.2).

(A) Western blot analysis of cellular and protein expression.

(B-G) Quantification of protein synthesis displayed in panel A $(n=3)$.

(B) Rictor/ß-actin; (C) phospho-Akt/Akt; (D) Raptor/ $\beta$-actin; (E) phospho-

S6K1/S6K1; (F) RSV nucleoprotein N/ $\beta$-actin; (G) RSV fusion protein $\mathrm{F} 1 / \beta$-actin.

Error bars, SEM; ${ }^{*}, p<0.05 ;{ }^{* \star}, p<0.01 ;{ }^{* \star *}, p<0.005 ;{ }^{* \star \star}, p<0.001$; n.s. non-significant.

\section{Knockdown of both Raptor and Rictor abolishes viral protein synthesis}

Given the redundancy of complex 1 and 2 activities during RSV replication, one would predict that knocking down both Raptor and Rictor simultaneously would impede viral protein synthesis (Figure 4A). Knockdown of Raptor in double shRictor/shRaptor cells abolished S6K1 activation, and reduced S6 phosphorylation (Figure 4B-D); whereas, knockdown of Rictor in double shRictor/shRaptor cells does not have much impact on Akt phosphorylation (Figure 4E-F), possibly due to more complete knocked down of Raptor than Rictor, which may resulted feed-back activation of Akt. However, knocking down both Rictor and Raptor within same cells abolished viral protein synthesis (Figure 4G-H). 

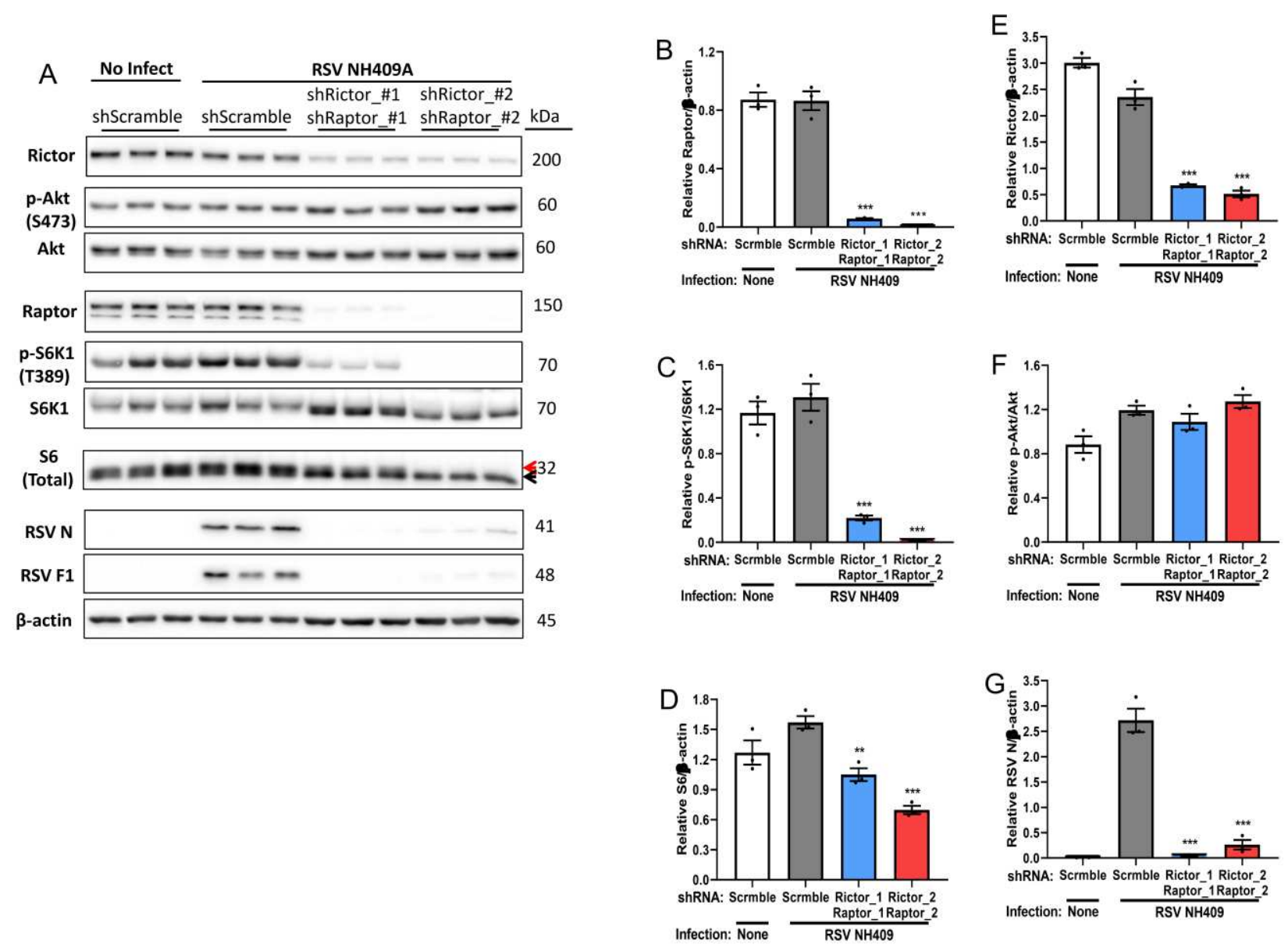

\section{Figure 4: Simultaneously knockdown both Raptor and Rictor block viral protein synthesis.}

Lenti-viral short-hairpin RNA (shRNA) were used to knockdown expression of both Raptor and Rictor in A549 cells. Double shRaptor/shRictor-A549 cells were infected with RSV NH409A. Cellular and viral protein expression was measures at 24 hour post infection (m.o.i $=0.2)$.

(A) Western blot analysis of cellular and protein expression.

(B-H) Quantification of protein synthesis displayed in panel A $(n=3)$.

(B) Raptor/ß-actin; (C) phospho-S6K1/S6K1; (D) total ribosomal protein S6/ßactin; (E) Rictor/ß-actin; (F) phospho-Akt/Akt; (G) RSV nucleoprotein N/ß-actin; (H) RSV fusion protein $F 1 / \beta$-actin.

Error bars, SEM; ${ }^{*}, p<0.05 ;{ }^{* *}, p<0.01 ;{ }^{* * *}, p<0.005 ;{ }^{* * *}, p<0.001$; n.s. non-significant. 


\section{Knockdown of mTOR kinase blocks viral protein synthesis}

mTORC1 is well-known to regulate cell growth and metabolism, while mTORC2 controls proliferation and survival ${ }^{19}$. Although they have different biological functions, these two distinct complexes shared the same mTOR kinase. To eliminate the possibility of differentially reduced levels of Raptor and Rictor in the gene knockdown experiment and a possible associated feed-back activation (Figure 4), mTOR was genetically knockdown by stable lenti-viral short-hairpin RNA (shRNA) (Figure 5A-B) ${ }^{37}$. Predictably, the knockdown of mTOR had minimal effect on Rictor and Raptor levels (Figure 5C-D), while there was significantly reduced Akt and S6K1 activation, and S6 phosphorylation (Figure $5 \mathrm{E}-\mathrm{G}$ ). Reduction of mTOR protein abolished viral protein synthesis (Figure $5 \mathrm{H}-\mathrm{I}$ ). Consistently, mTOR knockdown pheno-copied double Rictor/Raptor knockdown (Figure 4). Collectively, our data demonstrated the redundancy impact of each mTOR complex for RSV replication and the necessity of mTOR protein for viral protein expression. 

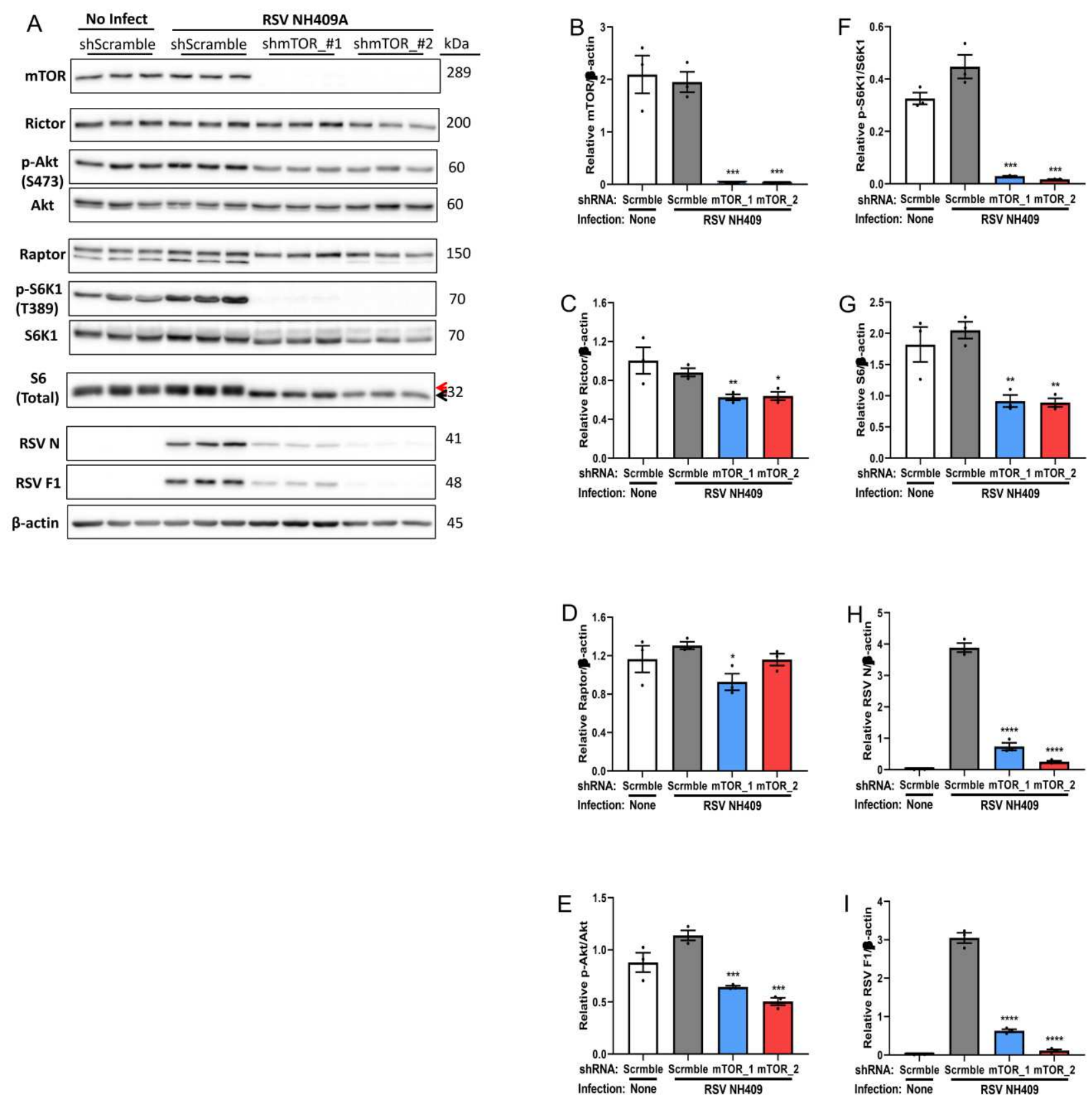

Figure 5: Genetic knockdown of mTOR kinase blocks viral protein synthesis. Lenti-viral short-hairpin RNA (shRNA) were used to knockdown expression of mTOR in A549 cells. shmTOR-A549 cells were infected with RSV NH409A. Cellular and viral protein expression was measures at 24 hour post infection (m.o.i $=0.2$ ).

(A) Western blot analysis of cellular and protein expression.

(B-I) Quantification of protein synthesis displayed in panel $A(n=3)$.

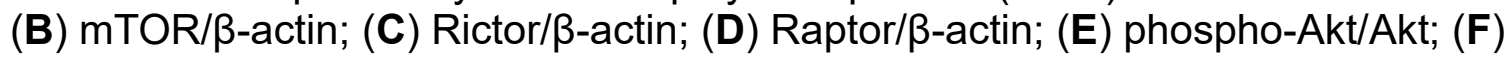
phospho-S6K1/S6K1; (G) total ribosomal protein S6/ $\beta$-actin; $(\mathbf{H}) \mathrm{RSV}$ nucleoprotein N/ $\beta$-actin; (I) RSV fusion protein $\mathrm{F} 1 / \beta$-actin.

Error bars, SEM; ${ }^{*}, p<0.05 ;{ }^{* *}, p<0.01 ;{ }^{* * *}, p<0.005 ;{ }^{* \star * *}, p<0.001$; n.s. non-significant. 


\section{Pharmacological interference of the function of mTOR complexes functions reduces RSV replication}

Although mTORC1 is the focal point of mTOR research, mTORC2 is emerging as an important complex in many cancers ${ }^{38,39}$. While there have been drugs developed to specifically target either mTORC1 alone or both complexes, drugs specific for mTORC2 have not yet been developed or validated for clinical use. To confirm the observations of mTOR knockdown (Figure 5) and potential therapeutic use, infected A549 cells were treated with dual complexes inhibitor AZD-8055, an ATP-competitive inhibitor of mTOR 40. Consistent with rapamycin, AZD-8055 treatment reduced phosphorylation of S6K1 and S6 (Figure 6A-C). In contrast to rapamycin however, AZD-8055 also reduced phosphorylation of Akt (Figure 6D). Provocatively, at $100 \mathrm{nM}$, AZD-8055 significantly reduced both viral protein synthesis and replication (Figure 6E-G). Similar observations could be seen with Torin-1, another ATP-competitive inhibitor of mTOR ${ }^{41}$ (Supplementary Figure 6). Our data suggesting mTOR kinase is a novel therapeutic target for RSV infection. 

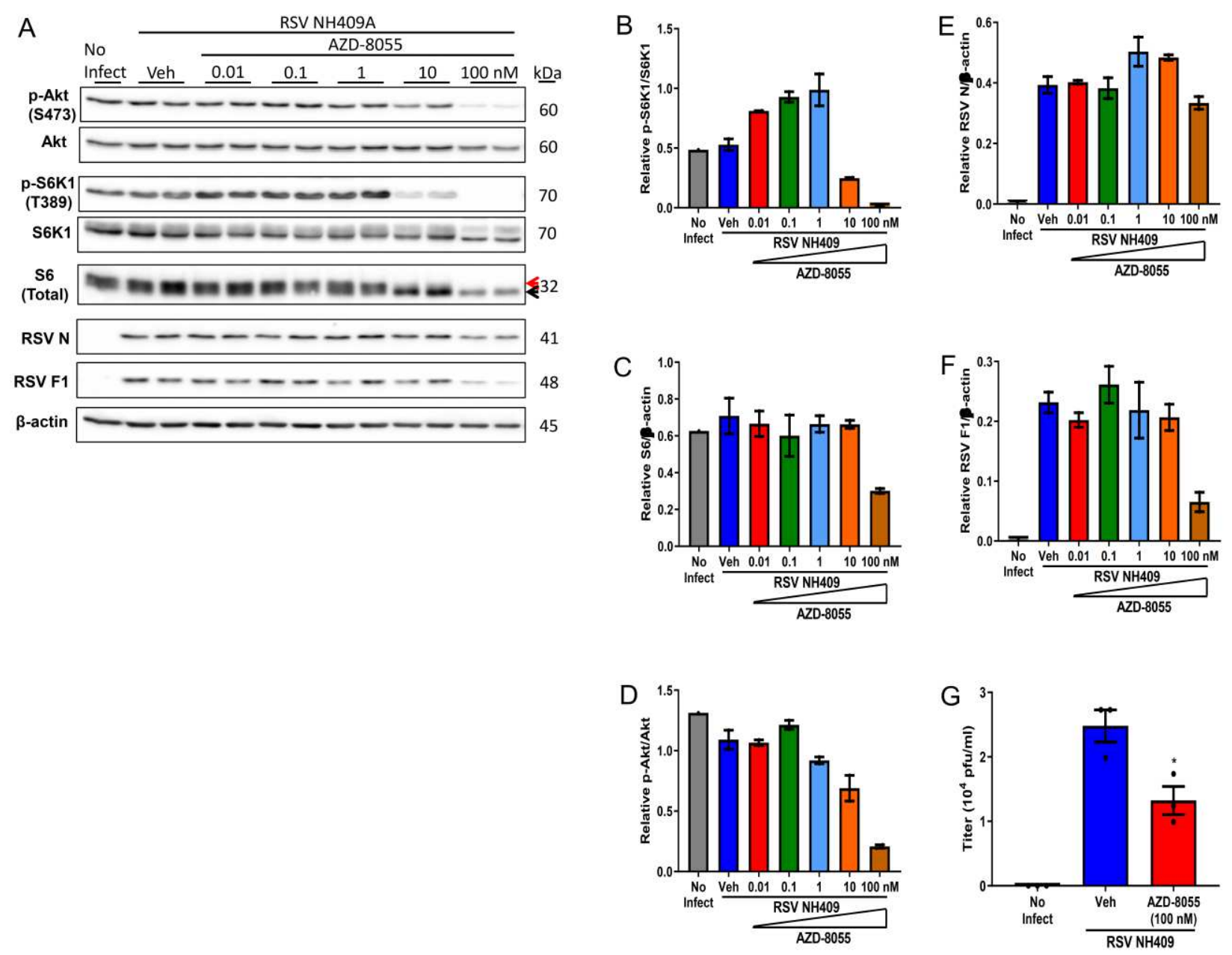

Figure 6: Inhibits both mTORC1 and mTORC2 by AZD-8055 Reduces viral protein expression and replication.

A549 cells were infected with clinical isolate $\mathrm{NH} 409 \mathrm{~A}$ and subsequently treated with varying concentrations of AZD-8055, an inhibitor of both mTORC1 and mTORC2. Protein analyses and viral replication was measured at 24 hours post infection (m.o.i = $0.2)$

(A) Western blot of cellular and viral proteins. The 2 forms of S6 are designated by the red and black arrows.

(B-F) Quantification of cellular and viral proteins displayed in $A(n=2)$.

(B) phospho-S6K1/S6K1; (C) total ribosomal protein S6/ $\beta$-actin; (D) phosphoAkt/Akt; (E) RSV nucleoprotein N/ $\beta$-actin; (F) RSV fusion protein F1/ $\beta$-actin.

(G) Quantification of virus progeny in the absence and presence of AZD-8055 $(n=3)$. Error bars, SEM; ${ }^{*}, p<0.05 ;{ }^{* *}, p<0.01 ;{ }^{* * *}, p<0.005 ;{ }^{* \star *}, p<0.001$; n.s. non-significant.

mTOR inhibitors block human coronavirus OC43 replication 
To test whether inhibiting the function of mTOR complexes would have negative impact on viral replication of beta-coronavirus, OC43-infected A549 cells were treated with various mTOR inhibitors (Figure 7). At 100 nM, both AZD-8055 (Figure 7A-B) and Torin1 (Figure 7C-D) abolished HCoV OC43 spike S protein synthesis. Similar observations could be seen at $1 \mu \mathrm{M}$ of Torkinib (PP 242), ATP-competitive mTOR inhibitor (Figure 7E-F) ${ }^{42,43}$. Inhibition of both Akt and S6K1 activation (phosphorylated) correlated with the abolishment of $S$ protein synthesis. The inhibition of viral protein $S$ correlated with a reduction in RNA expression, which was consistent across many viral genes (Figure 7GM, and Supplementary Figure 7A-G). Viral replication was also diminished (Figure 7N, and Supplementary Figure 8A-B). Together, our data showed that not only RSV, but also beta-coronavirus viral replication was inhibited by mTOR kinase inhibitors, suggesting a potential treatment for SARS-CoV-1, MERS, and SARS-CoV-2. 

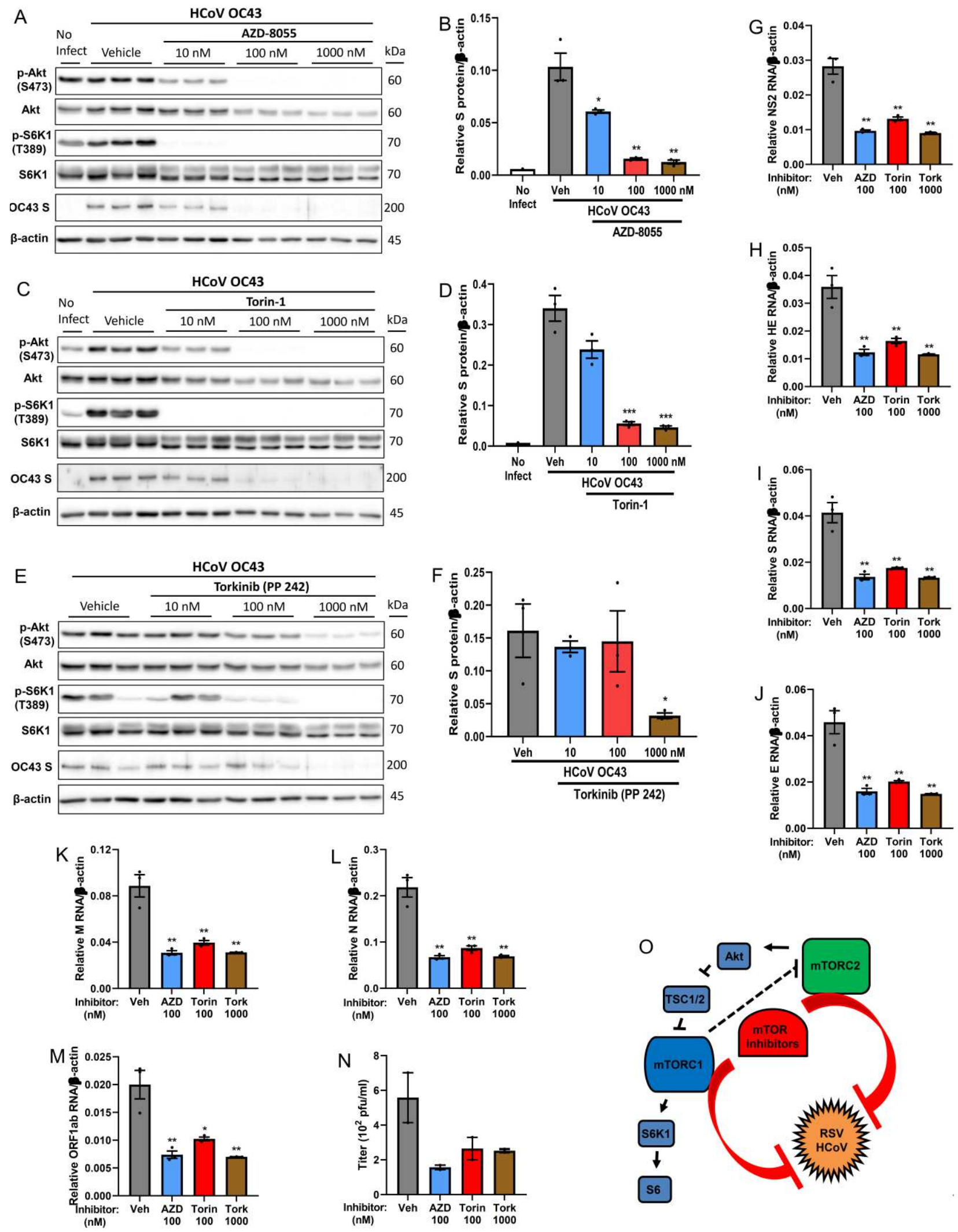

Figure 7: mTOR inhibitors inhibit human coronavirus OC43 replication. 
A549 cells were infected with human coronavirus (HCoV) OC43 for 1.5 hours and subsequently treated with different mTOR inhibitors, AZD-8055, Torin-1, or Torkinib, at varying concentrations. Protein $(\mathrm{MOI}=1)$, and viral RNA $(\mathrm{MOI}=0.3)$ analyses were measured at 24 hours post infection.

(A) Western blot of cellular and viral proteins at presence of AZD-8055.

(B) Quantification of viral protein spike S displayed in A $(n=3)$.

(C) Western blot of cellular and viral proteins at presence of Torin-1.

(D) Quantification of viral protein spike S displayed in C $(n=3)$.

(E) Western blot of cellular and viral proteins at presence of Torkinib.

(F) Quantification of viral protein spike S displayed in $E(n=3)$.

(G-M) Quantification of viral RNA expression at presence of indicated concentration of mTOR inhibitors $(n=3)$.

(G) non-structural protein 2 NS2; (H) hemagglutinin-esterase HE; (I) spike surface glycoprotein S; (J) envelope protein E; (K) membrane protein M; (L) nucleocapsid protein $\mathrm{N}$; $(\mathbf{M})$ replicase polyprotein ORF1ab.

(N) TCID50 quantification of virus progeny at presence of indicated concentration of mTOR inhibitors $(n=2)$.

(O) Schematic inhibition of RSV and HCoV. Simple illustration is adopted from previously review ${ }^{19}$. HCoV OC43 or RSV replications can be inhibited with mTOR inhibitors, including AZD-8055 and Torin-1.

Error bars, SEM; ${ }^{*}, p<0.05 ;{ }^{* *}, p<0.01 ;{ }^{* * *}, p<0.005 ;{ }^{* * *}, p<0.001$; n.s. non-significant.

\section{DISCUSSION}

Our studies further uncovered the biological importance of host/pathogen interactions during RSV infection and replication. Here, our data demonstrated that limiting either mTORC1 or mTORC2 signaling enhanced viral replication, while inhibiting both complexes impeded RSV protein synthesis, thereby establishing the requirement of mTOR signaling for productive RSV infection. Additionally, our studies highlighted the challenge of RSV infection in patients receiving chemotherapy or immunosuppressive agents such as mTOR inhibitors (as is the case in transplant recipients) as we have shown that these commonly used chemotherapeutic agents enhance RSV protein expression and viral replication in cell culture. Our observations are consistent with a previous study that showed that rapamycin increased RSV RNA in infected dendritic cells 
44. Moreover, a recent study showed that inhibition of mTORC1 by rapamycin enhanced autophagy and was associated with an increase of RSV replication in HEp-2 cells ${ }^{45}$, supporting our findings.

Rapamycin binds FKBP12 protein to form an mTORC1-specific inhibitor ${ }^{28-30}$. Rapamycin-FKBP12 complexes do not bind or inhibit mTORC2; however, prolonged rapamycin treatment does abrogate mTORC2 signaling, likely due to the inability of rapamycin-bound mTOR to incorporate into new mTORC2 complexes ${ }^{46,47}$. Consistence with this notion, high concentration of rapamycin treatment diminished or attenuated Akt phosphorylation (Supplementary Figure 2). Our data also showed significant induction of viral proteins and viral replication at high doses of rapamycin $(10-100 \mathrm{nM})$, which is equivalent to $9.14-91.4 \mathrm{ng} / \mathrm{mL}$. The target blood concentration of sirolimus, the FDA approved version of rapamycin, is $10-24 \mathrm{ng} / \mathrm{ml}{ }^{48-50}$ and therefore individuals who received sirolimus for immunosuppression (e.g. solid organ transplant recipients) or other indications may be at significant risk if they were to contract RSV infection.

Our data showed rapamycin increased viral replication, correlated with elevated Akt phosphorylation (Figure 1) and was confirmed by genetic knockdown of Raptor (Figure 3 \& Supplementary Figure 5), an indication of active mTORC2 signaling. Unfortunately, there are no validated drugs available to inhibit mTORC2 specifically. It is well known that mTORC2 is upstream of $\mathrm{mTORC} 1$, and $\mathrm{mTORC} 1$ negatively regulates autophagy ${ }^{19}$. By inhibiting both complexes at once via genetic knockdown of mTOR, one would expect to enhance autophagy ${ }^{51}$. If that were the case, then viral protein expression or viral replication should be increased. In contrast to our expectation, inhibiting both complexes with genetic knockdown of both Rictor and Raptor (Figure 4) or mTOR (Figure 5), 
confirmed by mTOR inhibitor AZD-8055 (Figure 6) decreased viral protein synthesis and replication. These observations are also in line with literature that autophagy is well known for its ability to limit intracellular pathogens replication ${ }^{52}$.

The inhibition of mTORC1 with rapamycin induced activation of mTORC2 (Akt phosphorylation) via the loss of negative feedback loops (Figure 1 \& Supplementary Figure 2) ${ }^{31,32}$. In line with those observations, genetic knockdown of Raptor (mTORC1) increased Akt phosphorylation (Figure 3 \& Supplementary Figure 5). Although mTORC1 is known to be a downstream target of mTORC2 (Figure 7G), one would expected inhibit mTORC2 function would diminish mTORC1 (S6K1 phosphorylation) signaling. However, knockdown of Rictor (mTORC2) resulted increase S6K1 phosphorylation. Previous studies have shown that mTORC2 appears to be necessary for Akt activity toward some but not all substrates ${ }^{37,53-56}$, suggesting the hyper-activation of S6K1 is possibly due to an unknown feedback mechanism. Knockdown of either Raptor or Rictor resulted in increased viral proteins, suggesting the mTOR complexes are redundant for RSV replication. To our knowledge, this is the first study to report this observation.

The first mTORC2 substrate identified was PKCa ${ }^{57,58}$. Recently, more mTORC2 targets were identified, including $\mathrm{PKC} \delta, \mathrm{PKC} \zeta, \mathrm{PKC} \gamma$, and $\mathrm{PKC} \varepsilon$ 59-61. More importantly, mTORC2 is well known for phosphorylation and activation of Akt ${ }^{37}$. Akt is known to promote cell survival, proliferation, and growth, and involved in many cancers ${ }^{19,35}$. Treatment with pan-Akt inhibitor MK-2206 modestly enhanced viral replication (Figure 2). Inhibition of Akt resulted slightly decreased of S6K1 phosphorylation (less active mTORC1), which possibly induced activation of mTORC2 via the loss of negative feedback loops. Our observations with MK-2206 have implications for other class of Akt 
inhibitors, specifically for individuals receiving related compounds for chemotherapy or immunosuppression who may encounter RSV.

In light of the current SARS-CoV-2 pandemic, we turned to explore the effects of targeting cellular functions on the replication of human coronaviruses. Effective prevention of COVID-19 required multifaceted efforts, including vaccinations and antiviral drugs. As of this writing, there are at least 6 vaccines that are in use in a variety of countries. These vaccines target the spike glycoprotein as neutralizing antibodies against this viral protein can prevent infection. However, it is now clear that mutations in the spike protein have emerged that alter its antigenic structure potentially limiting the specificity of the vaccineinduced immunity ${ }^{62,63}$. Further, there is concern that the new variants identified in the United Kingdom, South Africa, Brazil, and elsewhere have acquired the necessary mutations to make the vaccine-induced neutralizing antibodies ineffective ${ }^{64}$. There are a number of studies to determine whether any preexisting antiviral drugs have sufficient effectiveness for treating COVID-19 patients ${ }^{65,66}$. As of May 1, 2020, remdesivir, adenosine nucleotide triphosphate analog, has been authorized by U. S. Food and Drug Administration for compassionate use in the United States ${ }^{67}$. Due to the fact that SARSCoV-2 is prone to mutation, one foreseeable obstacle for antiviral therapy is the development of viral resistance, as viral genomic mutations occur during viral replication 17,18. Indeed, mutations in the RNA replicase of mouse hepatitis virus, a coronavirus, cause partial resistance to remdesivir ${ }^{68}$. Therefore, alternative therapeutic approaches should be considered. Once inside the host, coronaviruses utilize the host machinery for its replication. Here, we show that targeting mTOR kinase with various small molecule inhibitors is a means to inhibit or diminish viral protein synthesis or replication as we have 
learned from our RSV experiments. In parallel to RSV, treatment with mTOR inhibitors abolished or diminished HCoV OC43 spike protein synthesis and viral replication, providing a proof-of-concept for alternative therapeutics.

In summary, the significance and novelty of our current study include the following: 1) the dynamic utilization of mTOR signaling by RSV for its replication, established by pharmacologic and genetic models; 2) the redundancy of mTOR complexes for RSV replication, a novel finding; 3 ) the potential impact of mTOR and Akt inhibitors on RSV replication and the danger to cancer patients and others who are receiving these drugs if they would become infected with RSV or any of the beta-coronaviruses and ; 4) a proofof-concept for potential therapeutic treatment for SARS-CoV-1, MERS and SARS-CoV2. In all, the targeting of host factors may be a useful therapeutic strategy for existing or emerging human coronaviruses.

\section{EXPERIMENTAL PROCEDURES}

\section{Virus and cells}

RSV clinical isolates were obtained from RSV-infected individuals, as described previously, in New Haven, CT ${ }^{22}$ and Dallas, TX ${ }^{20}$. Collection and use of clinical isolates followed all institutional requirements and guidelines, were consistent with policies and regulations for the use of patient derived materials and approved by the respective Institutional Review Boards. The need to obtain informed consent was waived by the Institutional Review Board (IRB) of Yale University and the University of Texas Southwestern Medical Center. Isolates were plaque-purified, concentrated, quantified by 
plaque titration and working stocks prepared as described previously ${ }^{20}$. Viral stocks were prepared with a low inoculum ( $\mathrm{MOI}$ of 0.01$)$ to minimize the production of defective interfering (DI) particles. Further, the number of passages of virus in cell culture was limited to prevent the potential viral adaption to cell culture. For all experiments, a multiplicity of infection (MOI.) of 0.2 was used, otherwise stated. The m.o.i. was dependent upon the titers of the stocks of each of the clinical isolates. For each individual experiment, the m.o.i of each virus was identical. All specimens from which virus were obtained were submitted as part of routine care. Only left over material was used for viral propagation. Collection of specimens from the Clinical Virology Laboratory at Yale-New Haven Hospital was approved by the Yale University Human Investigations Committee. The RSV isolates from Dallas, Texas was propagated from a de-identified clinical specimen obtained from the Clinical Microbiology Laboratory at Children's Medical Center, Dallas. Collection and use of clinical isolates followed all institutional requirements and guidelines and was consistent with policies and regulations for the use of patient derived materials. Human coronavirus (HCoV) OC43 (ATCC VR-1558), and human HCT-8 colon cells (ATCC CCL-244) were obtained from American Type Culture Collection (Manassas, VA).

A549 (CCL-185) cells were obtained from ATCC and cultivated in F-12 Kaighn's modification media with $10 \%$ fetal bovine serum. Viral titers were determined using a plaque assay ${ }^{20}$. Plaques were detected with an immunohistochemical staining technique with primary anti-RSV Fusion protein antibody (palivizumab, Medlmmune, Gaithersberg, MD) and secondary HRP conjugated anti-human antibody (Jackson ImmunoResearch 
Laboratories, Inc., West grove, PA). HCoV OC43 viral titer was determined with HCT-8 cells by median tissue culture infectious dose (TCID50), according to ATCC protocol and briefly described elsewhere ${ }^{69}$.

\section{Chemicals, shRNA plasmids, and antibodies}

Rapamycin (LC Laboratories); MK-2206 HCl (Cayman Chemical); AZD-8055, Torin-1, and Torkinib (PP 242) (Medchemexpress) were dissolved in DMSO as manufacturer instructions. Equal volume of DMSO served as vehicle.

Scramble shRNA (1864), Raptor_1 shRNA (1857), Raptor_2 shRNA (1858), Rictor_1 shRNA (1853), Rictor_2shRNA (1854), mTOR_1shRNA (1855), mTOR_2 shRNA (1856)

plasmids gifted from David Sabatini ${ }^{37}$ were packaged by lentiviral plasmids pMD2.G (12259) psPAX2 (12260). pMD2.G and psPAX2 were gifts from Didier Trono. All of these plasmids above were obtained from Addgene. Briefly, shRNA plasmid together with pMD2.G and psPAX2 were transfected into 293T cells. Supernatants containing viruses were collected after 72 hours of transfection, and infected with A549 cells. A549 cells were screen with puromycin $(1.5 \mu \mathrm{g} / \mathrm{mL})$ after 3 days of infection. Western blots were performed to evaluate protein expression. All western blots/images are pre-cut.

Primary rabbit Phospho-Akt (S473), Akt, phospho-S6K1 (T389), S6K1, S6, mTOR, $\beta$ actin antibodies (Cell Signal Technology); rabbit Rictor and Raptor antibodies (Bethyl Laboratories); mouse RSV N antibody (Novus Biologicals); human RSV F1 (palivizumab) antibody (Medlmmune); rabbit coronavirus spike antibody (Invitrogen PA581777) were 
detected with secondary goat anti-rabbit HRP antibody (Thermo Fisher Scientific), horse anti-mouse HRP antibody (Cell Signal Technology), or rabbit anti-human HRP antibody (Jackson ImmunoResearch Laboratories).

\section{RSV and HCoV OC43 infection and protein detection}

$2 \times 10^{5}$ cells per well of 12 -well plate of $\mathrm{A} 549$ cells were infected, $\mathrm{MOI}$ of 0.2 , with sucrosepurified clinical isolates of RSV. After 90 minutes of infection, the inoculum was removed, the cells were washed with serum free media, and fresh F-12 Kaighn's modification media containing 5\% FBS with or without indicated drugs was added to the infected monolayers. The cells were directly lysed with laemmli sample buffer 24 hours post-infection. Western blotting was performed with SDS-PAGE gels. Chemiluminescence signals were captured by GE ImageQuant LAS-4000. Relative protein quantifications were analyzed by ImageJ 70. A549 cells were infected with $\mathrm{HCoV}$ OC43 at $\mathrm{MOI}$ of 1 , and western blotting was performed with SDS-PAGE gels 24 hours post-infection. All western blots/images are precut.

\section{RNA extraction, reverse transcription and quantitative PCR}

A549 cells were infected with $\mathrm{HCoV}$ OC43 at $\mathrm{MOI}$ of 0.3 . Total RNAs were extracted at 24 hours post-infection by TRIzol reagent (Invitrogen). First-strand of total cDNAs were synthesized with random primers by high capacity cDNA reverse transcription kit (Applied Biosystems). Quantitative PCR (qRT-PCR) was performed with PowerUp SYBR green (Applied Biosystems) and CFX96 thermal cycler (Bio-rad Laboratories). 


\section{Statistical Analyses}

All statistical analyses were performed with Student's t-test and represented as mean \pm S.E.M unless stated otherwise. The $p$ values were designated as ${ }^{*} p<0.05 ;{ }^{*} p<0.01$; ${ }^{* * *} p<0.005 ;{ }^{* * * *} p<0.001$; and n.s., nonsignificant $(p>0.05)$.

\section{REFERENCES}

1 Hall, C. B. Respiratory syncytial virus and parainfluenza virus. N Engl J Med 344, 1917-1928, doi:10.1056/NEJM200106213442507 (2001).

2 Nair, H. et al. Global burden of acute lower respiratory infections due to respiratory syncytial virus in young children: a systematic review and meta-analysis. Lancet 375, 1545-1555, doi:10.1016/s0140-6736(10)60206-1 (2010).

3 Anderson, L. J. et al. Antigenic characterization of respiratory syncytial virus strains with monoclonal antibodies. J Infect Dis 151, 626-633 (1985).

$4 \quad$ Peret, T. C. et al. Circulation patterns of group A and B human respiratory syncytial virus genotypes in 5 communities in North America. J Infect Dis 181, 1891-1896 (2000).

5 Collins, P. L., Hill, M. G., Cristina, J. \& Grosfeld, H. Transcription elongation factor of respiratory syncytial virus, a nonsegmented negative-strand RNA virus. Proc Natl Acad Sci U S A 93, 81-85, doi:10.1073/pnas.93.1.81 (1996).

6 Kuo, L., Fearns, R. \& Collins, P. L. The structurally diverse intergenic regions of respiratory syncytial virus do not modulate sequential transcription by a dicistronic minigenome. $J$ Virol 70, 6143-6150, doi:10.1128/JVI.70.9.6143-6150.1996 (1996).

7 Collins, P. L. et al. Production of infectious human respiratory syncytial virus from cloned cDNA confirms an essential role for the transcription elongation factor from the 5 ' proximal open reading frame of the $\mathrm{M} 2 \mathrm{mRNA}$ in gene expression and provides a capability for vaccine development. Proc Natl Acad Sci U S A 92, 11563-11567, doi:10.1073/pnas.92.25.11563 (1995).

8 Kahn, J. S., Schnell, M. J., Buonocore, L. \& Rose, J. K. Recombinant vesicular stomatitis virus expressing respiratory syncytial virus (RSV) glycoproteins: RSV fusion protein can mediate infection and cell fusion. Virology 254, 81-91, doi:10.1006/viro.1998.9535 (1999).

9 Kisch, A. L., Johnson, K. M. \& Chanock, R. M. Immunofluorescence with respiratory syncytial virus. Virology 16, 177-189, doi:10.1016/0042-6822(62)90293-3 (1962).

10 Follett, E. A., Pringle, C. R. \& Pennington, T. H. Virus development in enucleate cells: echovirus, poliovirus, pseudorabies virus, reovirus, respiratory syncytial virus and Semliki Forest virus. J Gen Virol 26, 183-196, doi:10.1099/0022-1317-26-2-183 (1975).

11 Chanock, R. \& Finberg, L. Recovery from infants with respiratory illness of a virus related to chimpanzee coryza agent (CCA). II. Epidemiologic aspects of infection in infants and young children. Am J Hyg 66, 291-300, doi:10.1093/oxfordjournals.aje.a119902 (1957). 

chimpanzees with coryza. Proc Soc Exp Biol Med 92, 544-549, doi:10.3181/00379727-92-22538 (1956).

13 Lieberthal, A. S. et al. Diagnosis and management of bronchiolitis. Pediatrics 118, 1774-1793, doi:10.1542/peds.2006-2223 (2006).

14 Halsey, N. A. et al. Prevention of respiratory syncytial virus infections: Indications for the use of palivizumab and update on the use of RSV-IGIV. Pediatrics 102, 1211-1216 (1998).

15 Fearns, R. \& Deval, J. New antiviral approaches for respiratory syncytial virus and other mononegaviruses: Inhibiting the RNA polymerase. Antiviral Res 134, 63-76, doi:10.1016/j.antiviral.2016.08.006 (2016).

16 Xing, Y. \& Proesmans, M. New therapies for acute RSV infections: where are we? Eur J Pediatr 178, 131-138, doi:10.1007/s00431-018-03310-7 (2019).

17 Agoti, C. N., Otieno, J. R., Gitahi, C. W., Cane, P. A. \& Nokes, D. J. Rapid Spread and Diversification of Respiratory Syncytial Virus Genotype ON1, Kenya. Emerg Infect Dis 20, 950959, doi:10.3201/eid2006.131438 (2014).

18 Griffiths, C., Drews, S. J. \& Marchant, D. J. Respiratory Syncytial Virus: Infection, Detection, and New Options for Prevention and Treatment. Clin Microbiol Rev 30, 277-319, doi:10.1128/CMR.00010-16 (2017).

19 Saxton, R. A. \& Sabatini, D. M. mTOR Signaling in Growth, Metabolism, and Disease. Cell 169, 361-371, doi:10.1016/j.cell.2017.03.035 (2017).

20 Levitz, R. et al. Induction of IL-6 and CCL5 (RANTES) in human respiratory epithelial (A549) cells by clinical isolates of respiratory syncytial virus is strain specific. Virol J 9, 190, doi:10.1186/1743422X-9-190 (2012).

21 Levitz, R. et al. Distinct patterns of innate immune activation by clinical isolates of respiratory syncytial virus. PLoS One 12, e0184318, doi:10.1371/journal.pone.0184318 (2017).

22 Martinello, R. A., Chen, M. D., Weibel, C. \& Kahn, J. S. Correlation between respiratory syncytial virus genotype and severity of illness. J Infect Dis 186, 839-842, doi:10.1086/342414 (2002).

23 Cui, J., Li, F. \& Shi, Z. L. Origin and evolution of pathogenic coronaviruses. Nat Rev Microbiol 17, 181-192, doi:10.1038/s41579-018-0118-9 (2019).

24 Kahn, J. S. \& Mclntosh, K. History and recent advances in coronavirus discovery. Pediatr Infect Dis J 24, S223-227, discussion S226, doi:10.1097/01.inf.0000188166.17324.60 (2005). Holmes, E. C. \& Rambaut, A. Viral evolution and the emergence of SARS coronavirus. Philos $T R$ Soc B 359, 1059-1065, doi:10.1098/rstb.2004.1478 (2004). Isolation of a Novel Coronavirus from a Man with Pneumonia in Saudi Arabia. New Engl J Med 367, 1814-1820, doi:10.1056/NEJMoa1211721 (2012).

27 Cohen, J. \& Normile, D. New SARS-like virus in China triggers alarm. Science 367, 234-235 (2020).

28 Brown, E. J. et al. A Mammalian Protein Targeted by G1-Arresting Rapamycin-Receptor Complex. Nature 369, 756-758, doi:DOI 10.1038/369756a0 (1994).

29 Sabatini, D. M., Erdjumentbromage, H., Lui, M., Tempst, P. \& Snyder, S. H. Raft1 - a Mammalian Protein That Binds to Fkbp12 in a Rapamycin-Dependent Fashion and Is Homologous to Yeast Tors. Cell 78, 35-43, doi:Doi 10.1016/0092-8674(94)90570-3 (1994).

30 Sabers, C. J. et al. Isolation of a Protein Target of the Fkbp12-Rapamycin Complex in Mammalian-Cells. Journal of Biological Chemistry 270, 815-822, doi:DOI 10.1074/jbc.270.2.815 (1995).

31 Hsu, P. P. et al. The mTOR-regulated phosphoproteome reveals a mechanism of mTORC1mediated inhibition of growth factor signaling. Science 332, 1317-1322, doi:10.1126/science.1199498 (2011). 
Yu, Y. H. et al. Phosphoproteomic Analysis Identifies Grb10 as an mTORC1 Substrate That Negatively Regulates Insulin Signaling. Science 332, 1322-1326, doi:10.1126/science.1199484 (2011).

33 Sparks, C. A. \& Guertin, D. A. Targeting mTOR: prospects for mTOR complex 2 inhibitors in cancer therapy. Oncogene 29, 3733-3744, doi:10.1038/onc.2010.139 (2010).

34 Cybulski, N. \& Hall, M. N. TOR complex 2: a signaling pathway of its own. Trends Biochem Sci 34, 620-627, doi:10.1016/j.tibs.2009.09.004 (2009).

35 Revathidevi, S. \& Munirajan, A. K. Akt in cancer: Mediator and more. Semin Cancer Biol 59, 8091, doi:10.1016/j.semcancer.2019.06.002 (2019). Hirai, H. et al. MK-2206, an allosteric Akt inhibitor, enhances antitumor efficacy by standard chemotherapeutic agents or molecular targeted drugs in vitro and in vivo. Mol Cancer Ther 9 , 1956-1967, doi:10.1158/1535-7163.MCT-09-1012 (2010).

37 Sarbassov, D. D., Guertin, D. A., Ali, S. M. \& Sabatini, D. M. Phosphorylation and regulation of Akt/PKB by the rictor-mTOR complex. Science 307, 1098-1101, doi:10.1126/science.1106148 (2005).

38 Magaway, C., Kim, E. \& Jacinto, E. Targeting mTOR and Metabolism in Cancer: Lessons and Innovations. Cells 8, doi:10.3390/cells8121584 (2019).

39 Gkountakos, A. et al. Unmasking the impact of Rictor in cancer: novel insights of mTORC2 complex. Carcinogenesis 39, 971-980, doi:10.1093/carcin/bgy086 (2018).

40 Chresta, C. M. et al. AZD8055 Is a Potent, Selective, and Orally Bioavailable ATP-Competitive Mammalian Target of Rapamycin Kinase Inhibitor with In vitro and In vivo Antitumor Activity. Cancer Res 70, 288-298, doi:10.1158/0008-5472.Can-09-1751 (2010).

41 Thoreen, C. C. et al. An ATP-competitive mammalian target of rapamycin inhibitor reveals rapamycin-resistant functions of mTORC1. J Biol Chem 284, 8023-8032, doi:10.1074/jbc.M900301200 (2009).

42 Apsel, B. et al. Targeted polypharmacology: discovery of dual inhibitors of tyrosine and phosphoinositide kinases. Nat Chem Biol 4, 691-699, doi:10.1038/nchembio.117 (2008).

43 Feldman, M. E. et al. Active-site inhibitors of mTOR target rapamycin-resistant outputs of mTORC1 and mTORC2. PLoS Biol 7, e38, doi:10.1371/journal.pbio.1000038 (2009). do Nascimento de Freitas, D., Gassen, R. B., Fazolo, T. \& Souza, A. P. D. Rapamycin increases RSV RNA levels and survival of RSV-infected dendritic cell depending on T cell contact. Toxicol In Vitro 36, 114-119, doi:10.1016/j.tiv.2016.07.016 (2016).

45 Li, M. et al. Respiratory Syncytial Virus Replication Is Promoted by Autophagy-Mediated Inhibition of Apoptosis. J Virol 92, doi:ARTN e02193-1710.1128/JVI.02193-17 (2018).

46 Lamming, D. W. et al. Rapamycin-Induced Insulin Resistance Is Mediated by mTORC2 Loss and Uncoupled from Longevity. Science 335, 1638-1643, doi:10.1126/science.1215135 (2012).

47 Sarbassov, D. D. et al. Prolonged rapamycin treatment inhibits mTORC2 assembly and Akt/PKB. Mol Cell 22, 159-168, doi:10.1016/j.molcel.2006.03.029 (2006).

48 Zhao, D. Q., Li, S. W. \& Sun, Q. Q. Sirolimus-Based Immunosuppressive Regimens in Renal Transplantation: A Systemic Review. Transplant Proc 48, 3-9, doi:10.1016/j.transproceed.2016.01.002 (2016).

49 Yakupoglu, Y. K. \& Kahan, B. D. Sirolimus: a current perspective. Exp Clin Transplant 1, 8-18 (2003).

50 Groth, C. G. et al. Sirolimus (rapamycin)-based therapy in human renal transplantation: similar efficacy and different toxicity compared with cyclosporine. Sirolimus European Renal Transplant Study Group. Transplantation 67, 1036-1042, doi:10.1097/00007890-199904150-00017 (1999).

51 Wang, X. C. et al. Knockdown of mTOR by lentivirus-mediated RNA interference suppresses atherosclerosis and stabilizes plaques via a decrease of macrophages by autophagy in 
apolipoprotein E-deficient mice. Int J Mol Med 32, 1215-1221, doi:10.3892/ijmm.2013.1494 (2013).

52 Levine, B. \& Kroemer, G. Biological Functions of Autophagy Genes: A Disease Perspective. Cell 176, 11-42, doi:10.1016/j.cell.2018.09.048 (2019).

53 Guertin, D. A. et al. Ablation in mice of the mTORC components raptor, rictor, or mLST8 reveals that mTORC2 is required for signaling to Akt-FOXO and PKC alpha but not S6K1. Dev Cell 11, 859871, doi:10.1016/j.devcel.2006.10.007 (2006).

54 Jacinto, E. et al. SIN1/MIP1 maintains rictor-mTOR complex integrity and regulates Akt phosphorylation and substrate specificity. Cell 127, 125-137, doi:DOI 10.1016/j.cell.2006.08.033 (2006).

55 Shiota, C., Woo, J. T., Lindner, J., Shelton, K. D. \& Magnuson, M. A. Multiallelic disruption of the rictor gene in mice reveals that mTOR complex 2 is essential for fetal growth and viability. Dev Cell 11, 583-589, doi:10.1016/j.devcel.2006.08.013 (2006). Yang, Q., Inoki, K., Ikenoue, T. \& Guan, K. L. Identification of $\operatorname{Sin} 1$ as an essential TORC2 component required for complex formation and kinase activity. Gene Dev 20, 2820-2832, doi:10.1101/gad.1461206 (2006).

57 Jacinto, E. et al. Mammalian TOR complex 2 controls the actin cytoskeleton and is rapamycin insensitive. Nat Cell Biol 6, 1122-U1130, doi:10.1038/ncb1183 (2004).

58 Sarbassov, D. D. et al. Rictor, a novel binding partner of mTOR, defines a rapamycin-insensitive and raptor-independent pathway that regulates the cytoskeleton. Curr Biol 14, 1296-1302, doi:DOI 10.1016/j.cub.2004.06.054 (2004).

59 Gan, X. Q. et al. PRR5L degradation promotes mTORC2-mediated PKC-delta phosphorylation and cell migration downstream of G alpha(12). Nat Cell Biol 14, 686-696, doi:10.1038/ncb2507 (2012).

$60 \mathrm{Li}, \mathrm{X}$. \& Gao, T. Y. mTORC2 phosphorylates protein kinase C zeta to regulate its stability and activity. Embo Rep 15, 191-198, doi:10.1002/embr.201338119 (2014).

61 Thomanetz, V. et al. Ablation of the mTORC2 component rictor in brain or Purkinje cells affects size and neuron morphology. J Cell Biol 201, 293-308, doi:10.1083/jcb.201205030 (2013).

62 Kupferschmidt, K. COVID-19 The pandemic virus is slowly mutating. But does it matter? Science 369, 238-239 (2020).

63 Dawood, A. A. Mutated COVID-19 may foretell a great risk for mankind in the future. New Microb New Infec 35, doi:ARTN 10067310.1016/j.nmni.2020.100673 (2020).

64 Garcia-Beltran, W. F. et al. Multiple SARS-CoV-2 variants escape neutralization by vaccineinduced humoral immunity. Cell, doi:10.1016/j.cell.2021.03.013 (2021).

65 Sarkar, C. et al. Potential Therapeutic Options for COVID-19: Current Status, Challenges, and Future Perspectives. Front Pharmacol 11, doi:ARTN 57287010.3389/fphar.2020.572870 (2020). Marouf, B. H. \& Dizaye, K. Re-tasking the use of pre-existing medications and potential therapeutic options for coronavirus disease (COVID-19): systematic review of clinical studies. Drug Discov Ther 14, 109-116, doi:10.5582/ddt.2020.03035 (2020).

67 Grein, J. et al. Compassionate Use of Remdesivir for Patients with Severe Covid-19. New Engl J Med 382, 2327-2336, doi:10.1056/NEJMoa2007016 (2020).

68 Agostini, M. L. et al. Coronavirus Susceptibility to the Antiviral Remdesivir (GS-5734) Is Mediated by the Viral Polymerase and the Proofreading Exoribonuclease. Mbio 9, doi:ARTN e002211810.1128/mBio.00221-18 (2018).

69 Ramakrishnan, M. A. Determination of $50 \%$ endpoint titer using a simple formula. World J Virol 5, 85-86, doi:10.5501/wjv.v5.i2.85 (2016).

70 Schneider, C. A., Rasband, W. S. \& Eliceiri, K. W. NIH Image to ImageJ: 25 years of image analysis. Nat Methods 9, 671-675, doi:10.1038/nmeth.2089 (2012). 
Figures

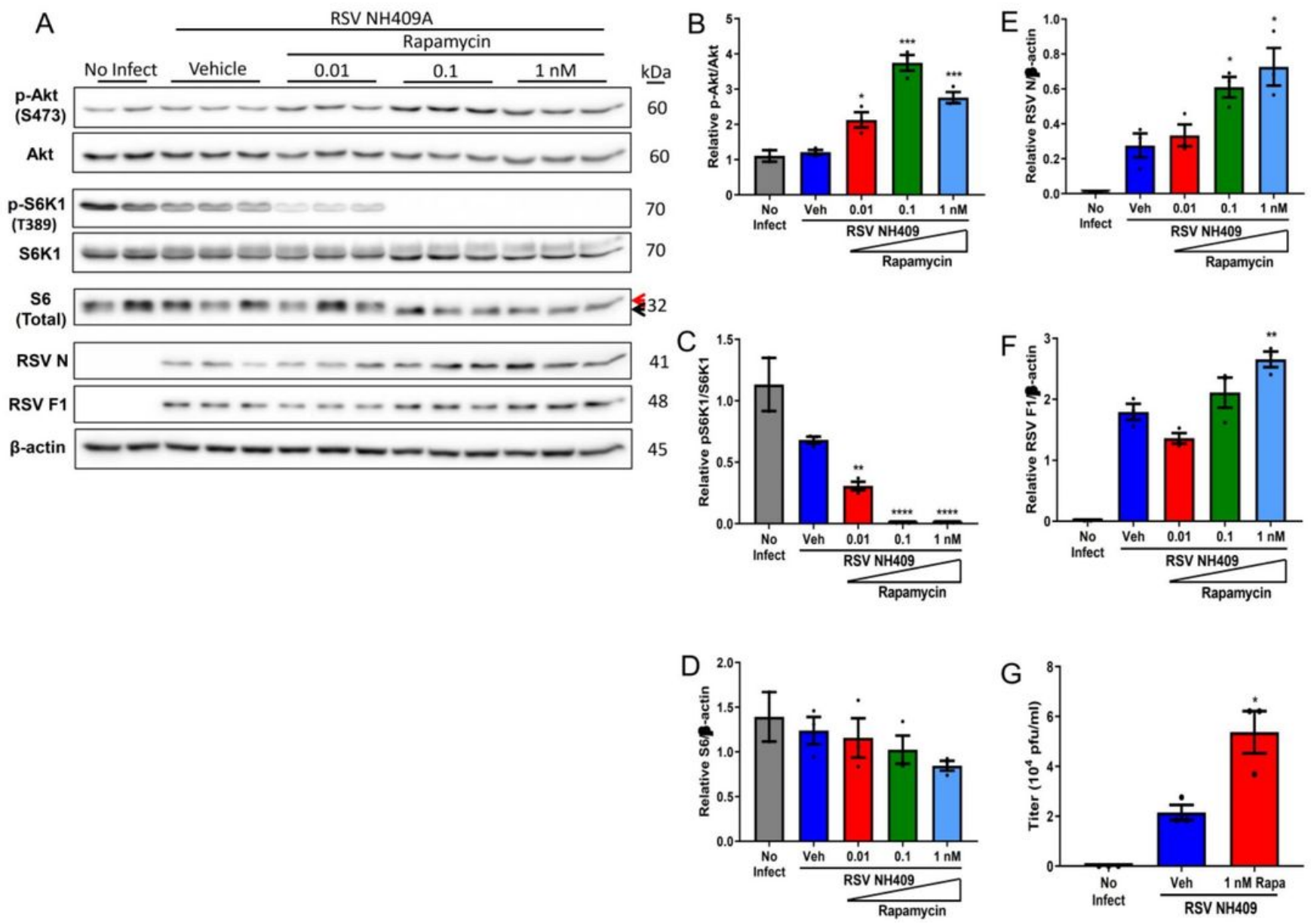

Figure 1

Please see the Manuscript PDF file for the complete figure caption 

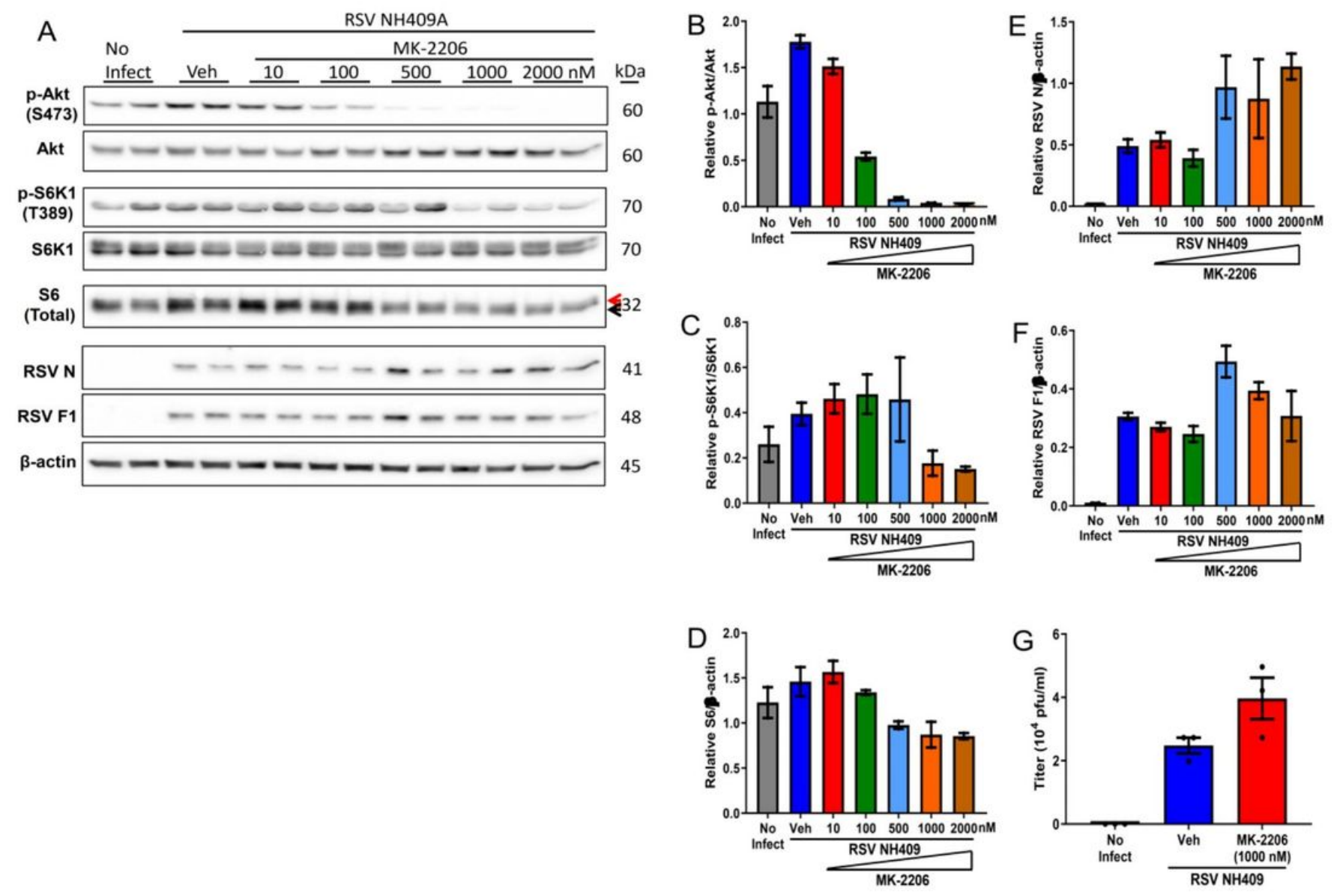

Figure 2

Please see the Manuscript PDF file for the complete figure caption 

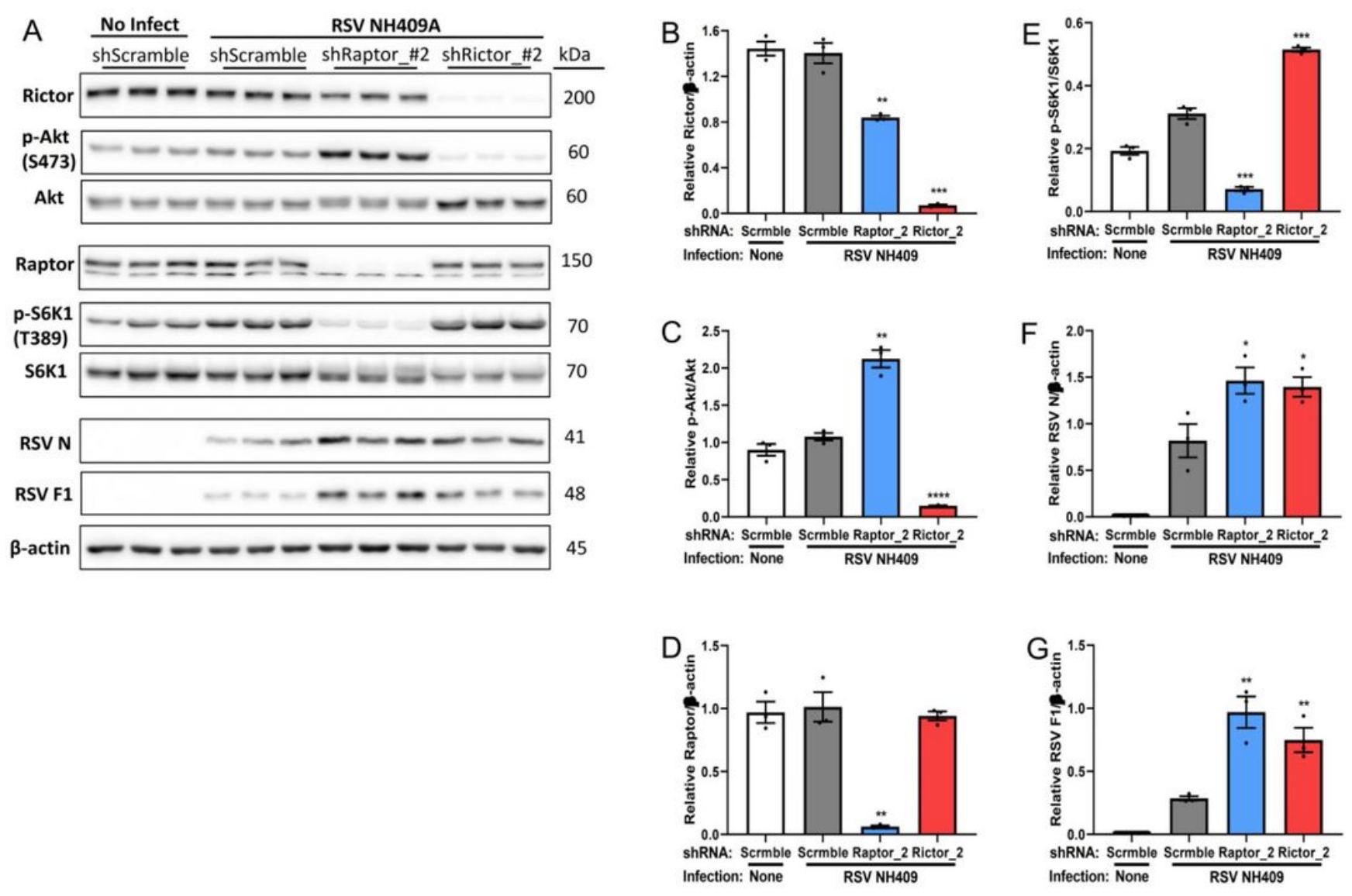

Figure 3

Please see the Manuscript PDF file for the complete figure caption 

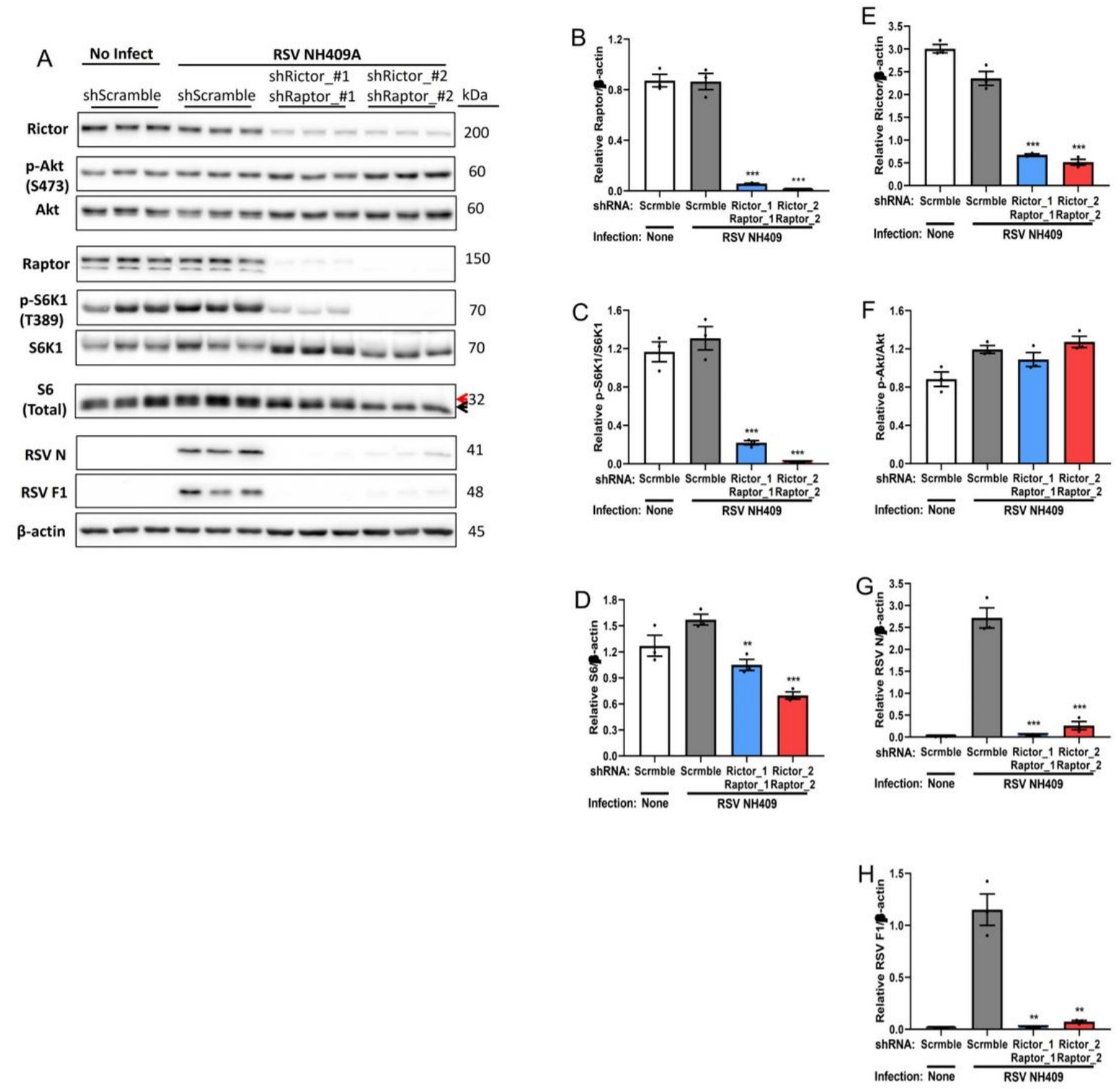

Figure 4

Please see the Manuscript PDF file for the complete figure caption 

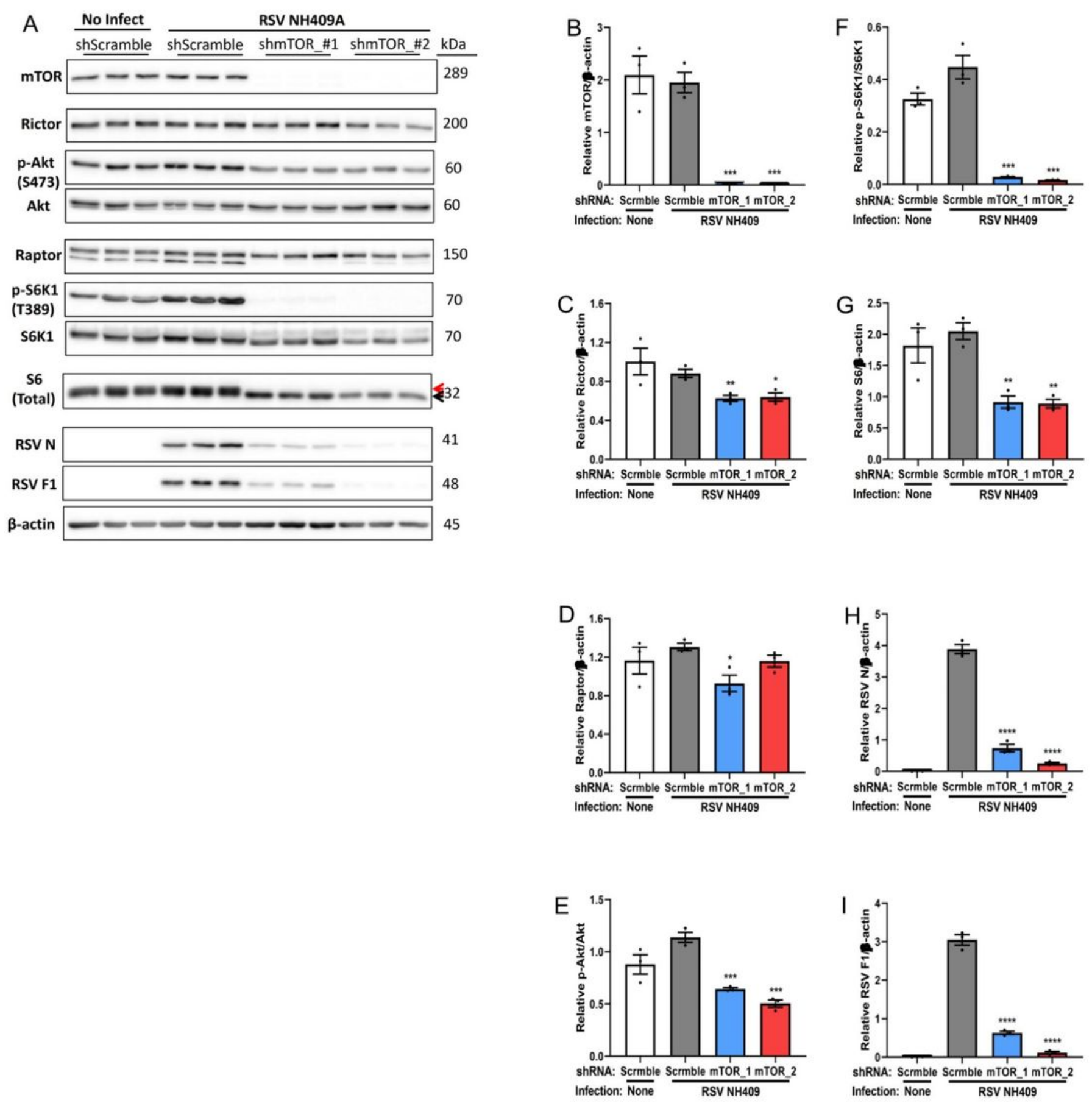

Figure 5

Please see the Manuscript PDF file for the complete figure caption 

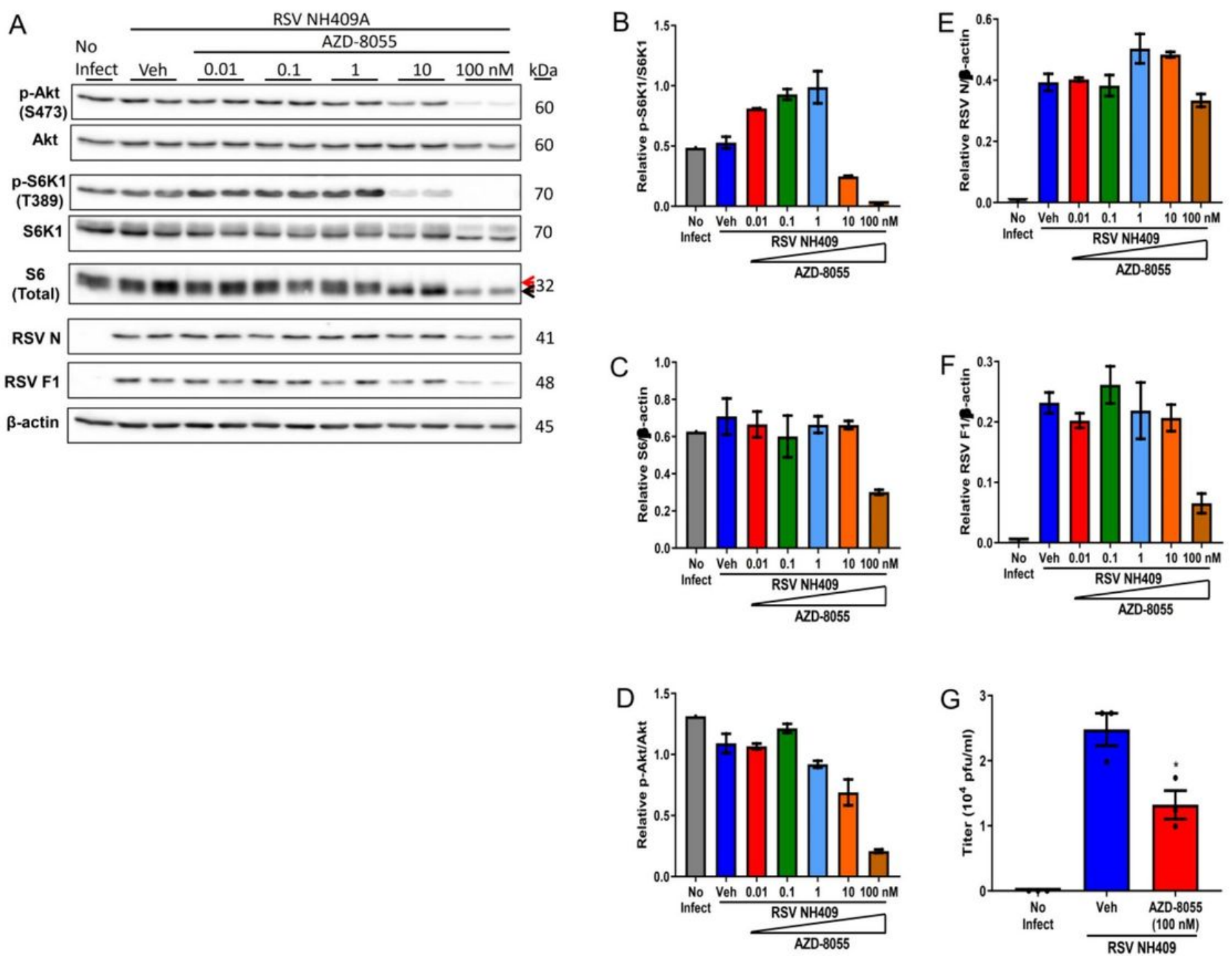

Figure 6

Please see the Manuscript PDF file for the complete figure caption 

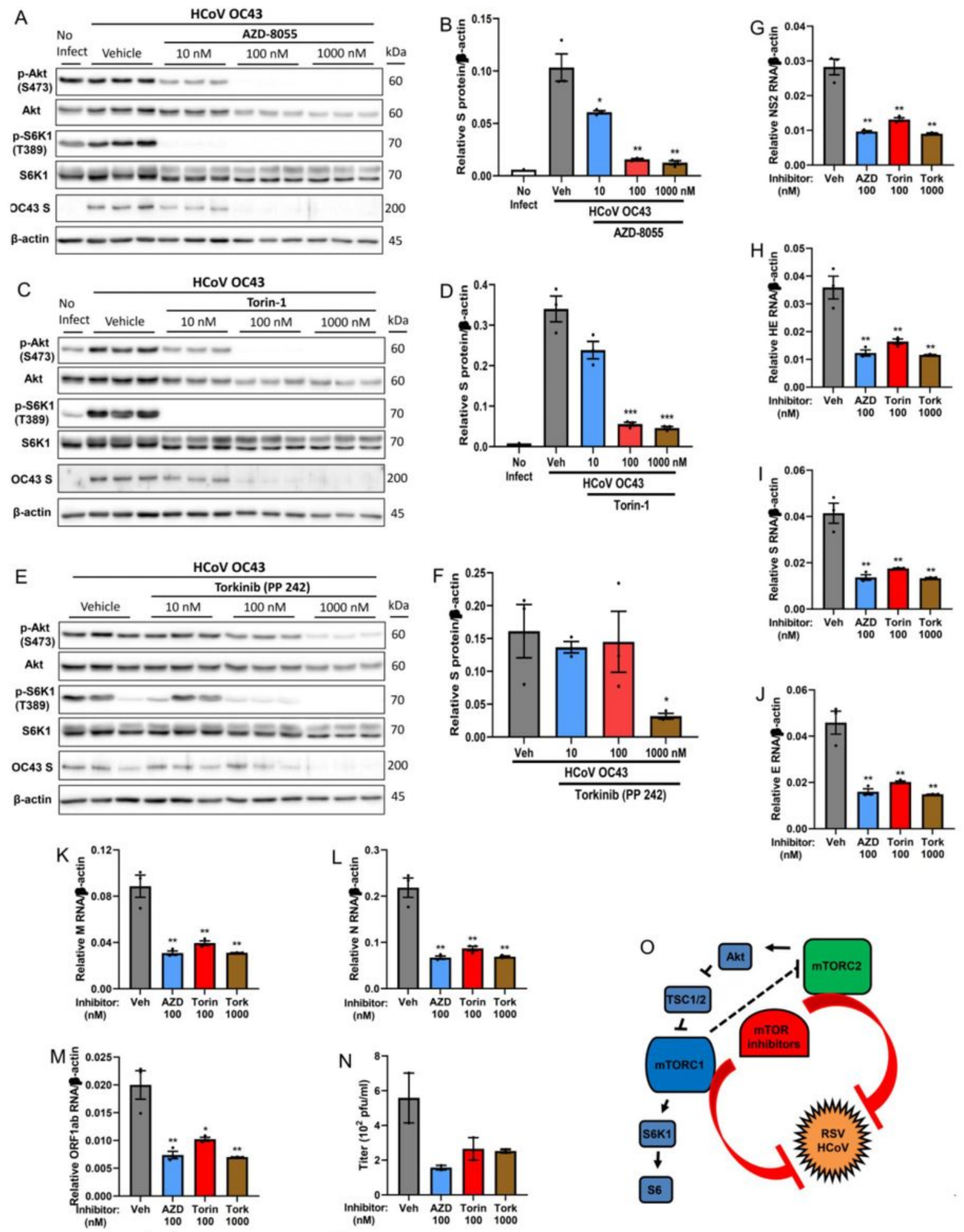

\section{Figure 7}

Please see the Manuscript PDF file for the complete figure caption

\section{Supplementary Files}

This is a list of supplementary files associated with this preprint. Click to download. 
- ScientificReportsSupplementaryFiguresandRawlmages52421.pdf 